\title{
Differing Averaged and Quenched Large Deviations for Random Walks in Random Environments in Dimensions Two and Three
}

\author{
Atilla Yilmaz $^{1, \star}$, Ofer Zeitouni ${ }^{1,2}$ \\ 1 Faculty of Mathematics, Weizmann Institute of Science, POB 26, Rehovot 76100, Israel \\ 2 School of Mathematics, University of Minnesota, Minneapolis, MN 55455, USA. \\ E-mail: zeitouni@math.umn.edu
}

Received: 8 October 2009 / Accepted: 6 April 2010

Published online: 29 August 2010 - (C) The Author(s) 2010. This article is published with open access at Springerlink.com

\begin{abstract}
We consider the quenched and the averaged (or annealed) large deviation rate functions $I_{q}$ and $I_{a}$ for space-time and (the usual) space-only RWRE on $\mathbb{Z}^{d}$. By Jensen's inequality, $I_{a} \leq I_{q}$. In the space-time case, when $d \geq 3+1, I_{q}$ and $I_{a}$ are known to be equal on an open set containing the typical velocity $\xi_{o}$. When $d=1+1$, we prove that $I_{q}$ and $I_{a}$ are equal only at $\xi_{o}$. Similarly, when $d=2+1$, we show that $I_{a}<I_{q}$ on a punctured neighborhood of $\xi_{o}$. In the space-only case, we provide a class of non-nestling walks on $\mathbb{Z}^{d}$ with $d=2$ or 3 , and prove that $I_{q}$ and $I_{a}$ are not identically equal on any open set containing $\xi_{o}$ whenever the walk is in that class. This is very different from the known results for non-nestling walks on $\mathbb{Z}^{d}$ with $d \geq 4$.
\end{abstract}

\section{Introduction}

1.1. The models. Consider a discrete time Markov chain on the $d$-dimensional integer lattice $\mathbb{Z}^{d}$ with $d \geq 1$. For any $x, z \in \mathbb{Z}^{d}$, denote the transition probability from $x$ to $x+z$ by $\pi(x, x+z)$. Refer to the transition vector $\omega_{x}:=(\pi(x, x+z))_{z \in \mathbb{Z}^{d}}$ as the environment at $x$. If the environment $\omega:=\left(\omega_{x}\right)_{x \in \mathbb{Z}^{d}}$ is sampled from a probability space $(\Omega, \mathcal{B}, \mathbb{P})$, then this process is called random walk in a random environment (RWRE). Here, $\mathcal{B}$ is the Borel $\sigma$-algebra corresponding to the product topology.

For every $y \in \mathbb{Z}^{d}$, define the shift $T_{y}$ on $\Omega$ by $\left(T_{y} \omega\right)_{x}:=\omega_{x+y}$. In order to have some statistical homogeneity in the environment, $\mathbb{P}$ is generally assumed to be stationary and ergodic with respect to $\left(T_{y}\right)_{y \in \mathbb{Z}^{d}}$. In this paper, we will make the stronger assumption that

$$
\mathbb{P} \text { is a product measure with equal marginals. }
$$

In other words, $\omega=\left(\omega_{x}\right)_{x \in \mathbb{Z}^{d}}$ is a collection of independent and identically distributed (i.i.d.) random vectors.

^ Current address: Department of Mathematics, University of California, Berkeley, CA 94720, USA. E-mail: atilla@math.berkeley.edu 
The set $\mathcal{R}:=\left\{z \in \mathbb{Z}^{d}: \mathbb{P}(\pi(0, z)>0)>0\right\}$ is the range of allowed steps of the walk (here and throughout, we often use 0 to denote the origin in $\mathbb{Z}^{d}$ when no confusion occurs). Let $\left(e_{i}\right)_{i=1}^{d}$ denote the canonical basis for $\mathbb{Z}^{d}$. The walk is said to be space-time if

$$
\mathcal{R}=\mathcal{R}_{s t}:=\left\{\left(z_{1}, \ldots, z_{d}\right) \in \mathbb{Z}^{d}:\left|z_{1}\right|+\cdots+\left|z_{d-1}\right|=1, z_{d}=1\right\},
$$

and it is said to be space-only if

$$
\mathcal{R}=\mathcal{R}_{\text {so }}:=\left\{ \pm e_{i}\right\}_{i=1}^{d} .
$$

In either case, we will assume throughout the paper that there exists a $\kappa>0$ such that $\mathbb{P}(\pi(0, z) \geq \kappa)=1$ for every $z \in \mathcal{R}$. This condition is known as uniform ellipticity.

Space-time is a natural term for the case (1.2) since then, the walk decomposes into two parts. Its projection on the $e_{d}$-axis is deterministic and can be identified with time. The motion in the span of $\left(e_{i}\right)_{i=1}^{d-1}$ can be thought of as a variation of space-only RWRE where the environment is freshly sampled at each time step. To emphasize this decomposition, we will write the dimension as $d=(d-1)+1$. For example, when $d=3$, we will say that the dimension is $2+1$.

For every $x \in \mathbb{Z}^{d}$ and $\omega \in \Omega$, the Markov chain with environment $\omega$ induces a probability measure $P_{x}^{\omega}$ on the space of paths starting at $x$. Statements about $P_{x}^{\omega}$ that hold for $\mathbb{P}$-a.e. $\omega$ are referred to as quenched. Statements about the semi-direct product $P_{x}:=\mathbb{P} \times P_{x}^{\omega}$ are referred to as averaged (or annealed). Expectations under $\mathbb{P}, P_{x}^{\omega}$ and $P_{x}$ are denoted by $\mathbb{E}, E_{x}^{\omega}$ and $E_{x}$, respectively.

See [25] for a survey of results and open problems on RWRE.

It is clear that no model satisfies both (1.2) and (1.3). Nevertheless, it turns out that many of the results that hold for space-only RWRE are valid also under the space-time assumption, and it is fair to say that space-time RWRE is easier to analyze than spaceonly RWRE because (1.2) ensures that the walk never visits the same point more than once.

1.2. Regeneration times. In the next subsection, we will give a brief survey of the previous results on large deviations for RWRE in order to put the present work in context. Some of these results involve certain random times which are introduced below for convenience.

Let $\left(X_{n}\right)_{n \geq 0}$ denote the path of a space-only RWRE. Consider a unit vector $\hat{u} \in \mathcal{S}^{d-1}$. Define a sequence $\left(\tau_{m}\right)_{m \geq 0}$ of random times, which are referred to as regeneration times (relative to $\hat{u}$ ), by $\tau_{o}:=\overline{0}$ and

$$
\tau_{m}:=\inf \left\{j>\tau_{m-1}:\left\langle X_{i}, \hat{u}\right\rangle<\left\langle X_{j}, \hat{u}\right\rangle \leq\left\langle X_{k}, \hat{u}\right\rangle \text { for all } i, k \text { with } i<j<k\right\}
$$

for every $m \geq 1$. (Regeneration times first appeared in the work of Kesten [9] on onedimensional RWRE. They were adapted to the multidimensional setting by Sznitman and Zerner, cf. [18].) Because we assumed the environment $\omega=\left(\omega_{x}\right)_{x \in \mathbb{Z}^{d}}$ to be an i.i.d. collection, if the walk is directionally transient relative to $\hat{u}$, i.e., if $P_{o}\left(\lim _{n \rightarrow \infty}\left\langle X_{n}, \hat{u}\right\rangle=\right.$ $\infty)=1$, then $P_{o}\left(\tau_{m}<\infty\right)=1$ for every $m \geq 1$. In this setup, as noted in [18], the significance of $\left(\tau_{m}\right)_{m \geq 1}$ is due to the fact that

$$
\left(X_{\tau_{m}+1}-X_{\tau_{m}}, X_{\tau_{m}+2}-X_{\tau_{m}}, \ldots, X_{\tau_{m+1}}-X_{\tau_{m}}, \tau_{m+1}-\tau_{m}\right)_{m \geq 1}
$$

is an i.i.d. sequence under $P_{o}$. 
The walk is said to satisfy Sznitman's transience condition (T) if

$$
E_{o}\left[\sup _{1 \leq i \leq \tau_{1}} \exp \left\{c_{1}\left|X_{i}\right|\right\}\right]<\infty \text { for some } c_{1}>0 .
$$

(Here and throughout, the norm $|\cdot|$ denotes the $\ell_{2}$ norm.) When $d \geq 2$, Sznitman [17] proves that (1.1), (1.3) and (T) imply a ballistic law of large numbers (LLN), an averaged central limit theorem and certain large deviation estimates.

Condition (T) holds as soon as the walk is non-nestling relative to $\hat{u}$, i.e., when the random drift vector

$$
v(\omega):=\sum_{z \in \mathcal{R}} \pi(0, z) z \quad \text { satisfies } \quad \operatorname{ess} \inf _{\mathbb{P}}\langle v(\cdot), \hat{u}\rangle>0 .
$$

The walk is said to be non-nestling if it is non-nestling relative to some unit vector. Otherwise, it is referred to as nestling. In the latter case, the convex hull of the support of the law of $v(\cdot)$ contains the origin.

In the case of space-time RWRE, regeneration times are defined naturally by taking $\hat{u}=e_{d}$ and $\tau_{m}=m$ for every $m \geq 1$. Clearly, the space-time walk is always non-nestling relative to $\hat{u}=e_{d}$.

1.3. Previous results on large deviations for $R W R E$. Recall that a sequence $\left(Q_{n}\right)_{n \geq 1}$ of probability measures on a topological space $\mathbb{X}$ is said to satisfy the large deviation principle (LDP) with a rate function $I: \mathbb{X} \rightarrow \mathbb{R}_{+} \cup\{\infty\}$ if $I$ is lower semicontinuous and for any measurable set $G$,

$$
-\inf _{x \in G^{o}} I(x) \leq \liminf _{n \rightarrow \infty} \frac{1}{n} \log Q_{n}(G) \leq \limsup _{n \rightarrow \infty} \frac{1}{n} \log Q_{n}(G) \leq-\inf _{x \in \bar{G}} I(x) .
$$

Here, $G^{o}$ is the interior of $G$, and $\bar{G}$ its closure. See [4] for general background regarding large deviations.

We will focus on the following large deviation principles for walks in uniformly elliptic environments.

Theorem 1.1 (Quenched LDP). For $\mathbb{P}$-a.e. $\omega,\left(P_{o}^{\omega}\left(\frac{X_{n}}{n} \in \cdot\right)\right)_{n \geq 1}$ satisfies the LDP with a deterministic and convex rate function $I_{q}$.

Theorem 1.2 (Averaged LDP). $\left(P_{o}\left(\frac{X_{n}}{n} \in \cdot\right)\right)_{n \geq 1}$ satisfies the LDP with a convex rate function $I_{a}$.

There are many works on large deviations for space-only RWRE. We briefly mention them in chronological order. Greven and den Hollander [7] prove Theorem 1.1 for walks on $\mathbb{Z}$ under the i.i.d. environment assumption. They provide a formula for $I_{q}$ and show that its graph typically has flat pieces. Zerner [26] establishes Theorem 1.1 for nestling walks on $\mathbb{Z}^{d}$ in i.i.d. environments. Comets, Gantert and Zeitouni [3] generalize the result of [7] to walks on $\mathbb{Z}$ in stationary and ergodic environments. Also, they prove Theorem 1.2 for walks on $\mathbb{Z}$ in i.i.d. environments and give a formula that links $I_{a}$ to $I_{q}$. Varadhan [20] generalizes Zerner's result to stationary and ergodic environments without any nestling assumption. He also proves Theorem 1.2 for walks on $\mathbb{Z}^{d}$ in i.i.d. 
environments and gives a variational formula for $I_{a}$. Rassoul-Agha [12] generalizes the latter result of [20] to certain mixing environments. Rosenbluth [15] gives an alternative proof of Theorem 1.1 for walks on $\mathbb{Z}^{d}$ in stationary and ergodic environments, and provides a variational formula for $I_{q}$. Yilmaz [23] generalizes the result of [15] to a so-called level-2 LDP. Berger [1], Peterson and Zeitouni [11], and Yilmaz [21] obtain certain qualitative properties of $I_{a}$. Rassoul-Agha and Seppäläinen [14] generalize the result of [15] to a so-called level-3 LDP.

In the case of space-time RWRE, Rassoul-Agha and Seppäläinen [13] prove Theorem 1.1 by adapting the quenched argument in [20]. Theorem 1.2 does not require any work. Indeed, Assumption (1.2) implies that the walk under $P_{o}$ is a sum of i.i.d. increments. The common distribution of these increments is $(q(z))_{z \in \mathcal{R}}$, where $q(z):=\mathbb{E}[\pi(0, z)]$ for every $z \in \mathcal{R}$. Therefore, Theorem 1.2 in the space-time setup is simply Cramér's Theorem, cf. [4].

In addition to the works mentioned in the last two paragraphs, there are two more results on large deviations for RWRE that are relevant to this paper. We state them in detail.

Theorem 1.3 (Yilmaz [22]). Assume (1.1) and (1.2). If $d \geq 3+1$, then $I_{q}=I_{a}$ on a set $\mathcal{A}_{s t} \times\left\{e_{d}\right\}$ containing the $L L N$ velocity $\xi_{o}$, where $\mathcal{A}_{s t}$ is an open subset of $\mathbb{R}^{d-1}$.

Theorem 1.4 (Yilmaz [24]). Assume (1.1), (1.3), $d \geq 4$, and that Sznitman's (T) condition holds for some $\hat{u} \in \mathcal{S}^{d-1}$.

(a) If the walk is non-nestling, then $I_{q}=I_{a}$ on an open set $\mathcal{A}_{\text {so }}$ containing the LLN velocity $\xi_{o}$.

(b) If the walk is nestling, then

(i) $I_{q}=I_{a}$ on an open set $\mathcal{A}_{\text {so }}^{+}$,

(ii) there exists a $(d-1)$-dimensional smooth surface patch $\mathcal{A}_{\text {so }}^{b}$ such that $\xi_{o} \in$ $\mathcal{A}_{\text {so }}^{b} \subset \partial \mathcal{A}_{\text {so }}^{+}$,

(iii) the unit vector $\eta_{o}$ normal to $\mathcal{A}_{\text {so }}^{b}$ (and pointing inside $\mathcal{A}_{\text {so }}^{+}$) at $\xi_{\text {o }}$ satisfies $\left\langle\eta_{o}, \xi_{o}\right\rangle>0$, and

(iv) $I_{q}(t \xi)=t I_{q}(\xi)=t I_{a}(\xi)=I_{a}(t \xi)$ for every $\xi \in \mathcal{A}_{\text {so }}^{b}$ and $t \in[0,1]$.

It is worthwhile to emphasize that the equality $I_{q}=I_{a}$ does not extend, in the setup of Theorems 1.3 and 1.4, to the whole space. Indeed, for any $d \geq 1$,

$$
I_{a}<I_{q} \text { at the extremal points of the domain of } I_{a} .
$$

By continuity, this inequality holds also at some interior points. See Proposition 4 of [24] for details.

1.4. Our results. For space-time RWRE, it is natural to ask whether Theorem 1.3 can be generalized to $d \geq 1+1$ or $2+1$. The answer turns out to be no.

Theorem 1.5. Assume (1.1) and (1.2). If $d=1+1$, then $I_{q}(\xi)=I_{a}(\xi)<\infty$ if and only if $\xi=\xi_{o}$, the $L L N$ velocity.

Theorem 1.6. Assume (1.1) and (1.2). Ifd $=2+1$, then $I_{a}<I_{q}$ on a set $\left(\mathcal{G}_{s t} \times\left\{e_{3}\right\}\right) \backslash\left\{\xi_{o}\right\}$, where $\mathcal{G}_{s t} \subset \mathbb{R}^{2}$ is open and $\mathcal{G}_{\text {st }} \times\left\{e_{3}\right\}$ contains $\xi_{o}$. 
In the case of space-only RWRE on $\mathbb{Z}$, a consequence of Comets et al. [3], Proposition 5 , is that $I_{q}(\xi)=I_{a}(\xi)<\infty$ if and only if $\xi=0$ or $I_{a}(\xi)=0$. In particular, Theorem 1.4 cannot be generalized to $d \geq 1$. Our next result shows that the conclusion of Theorem 1.4 is false for a class of space-only RWRE's in dimensions $d=2,3$.

Definition 1.7. Assume $d \geq 2$, and fix a triple $p=\left(p^{+}, p^{o}, p^{-}\right)$of positive real numbers such that $p^{-}<p^{+}$and $p^{+}+p^{o}+p^{-}=1$. For any $\epsilon>0$, a probability measure $\mathbb{P}$ on $(\Omega, \mathcal{B})$ is said to be in class $\mathcal{M}_{\epsilon}(d, p)$ if

(a) (1.1) and (1.3) hold,

(b) $\mathbb{P}\left(\pi\left(0, e_{d}\right)=p^{+}, \pi\left(0,-e_{d}\right)=p^{-}\right)=1$,

(c) $\mathbb{P}\left(\epsilon / 2<\left|\pi\left(0, e_{1}\right)-\frac{p^{o}}{2(d-1)}\right|<\epsilon\right)=1$, and

(d) $\mathbb{P}$ is invariant under the rotations of $\mathbb{Z}^{d}$ that preserve $e_{d}$. (We will refer to this as isotropy.)

Theorem 1.8. Assume $d=2$ or 3. Fix a triple $p=\left(p^{+}, p^{o}, p^{-}\right)$as in Definition 1.7. Then there exists an $\epsilon_{o}=\epsilon_{o}(p)$ such that if $\epsilon<\epsilon_{o}$ and $\mathbb{P}$ is in class $\mathcal{M}_{\epsilon}(d, p)$, then the quenched and the averaged rate functions $I_{q}$ and $I_{a}$ are not identically equal on any open set containing the LLN velocity $\xi_{o}$.

The proofs of our results are based on a technique that combines the so-called fractional moment method with a certain change of measure (which we will refer to as tilting the environment). This technique has been developed for analyzing the so-called polymer pinning model, cf. [5,6,19], and it has been recently refined by Lacoin [10] for obtaining certain lower bounds for the free energy of directed polymers in random environments. Comparing with the polymer setup, an extra complication occurs in the RWRE model due to the dependence of the transition probabilities of the walk on the environment. (In the polymer model discussed above, the walk is a simple random walk, and the environment only appears in the evaluation of exponential moments with respect to the random walk.) The difficulty in the RWRE setup, and much of our work, lies in overcoming this dependency. For space-time RWRE, this task is greatly simplified because each site is visited at most once. For space-only RWRE, where this is not true, we employ a perturbative approach that unfortunately restricts the class of models considered, see Sect. 4 for further comments.

Here is how the rest of the paper is organized: In Sect. 2, we consider space-time RWRE and prove Theorems 1.5 and 1.6 by adapting the relevant arguments given in [10]. In Sect. 3, we focus on space-only walks that are non-nestling relative to $e_{d}$, and modify the previous proofs by making use of regeneration times. This way, we establish a result (see Theorem 3.4) analogous to Theorems 1.5 and 1.6. The only difference is that Theorem 3.4 is valid under a certain correlation condition, cf. (3.17). Finally, we prove Theorem 1.8 by checking that (3.17) holds whenever $\mathbb{P}$ is in class $\mathcal{M}_{\epsilon}(d, p)$ with some triple $p$ (as in Definition 1.7) and a sufficiently small $\epsilon>0$.

\section{Inequality of the Rate Functions for Space-Time RWRE}

2.1. Reducing to a fractional moment estimate. Assume $d \geq 1+1$. Recall (1.2). Consider a space-time random walk on $\mathbb{Z}^{d}$ in a uniformly elliptic and i.i.d. environment. For every $\theta \in \mathbb{R}^{d}$, define

$$
\phi(\theta):=\sum_{z \in \mathcal{R}} \mathrm{e}^{\langle\theta, z\rangle} q(z)
$$


where $q(z):=\mathbb{E}[\pi(0, z)]$. Since the walk visits every point at most once, $E_{o}[\exp$ $\left.\left\{\left\langle\theta, X_{N}\right\rangle\right\}\right]=\phi(\theta)^{N}$ for every $N \geq 1$.

Define the logarithmic moment generating functions

$$
\begin{aligned}
& \Lambda_{q}(\theta):=\lim _{N \rightarrow \infty} \frac{1}{N} \log E_{o}^{\omega}\left[\exp \left\{\left\langle\theta, X_{N}\right\rangle\right\}\right] \text { and } \\
& \Lambda_{a}(\theta):=\lim _{N \rightarrow \infty} \frac{1}{N} \log E_{o}\left[\exp \left\{\left\langle\theta, X_{N}\right\rangle\right\}\right]=\log \phi(\theta) .
\end{aligned}
$$

By Varadhan's Lemma, cf. [4], $\Lambda_{q}(\theta)=\sup _{\xi \in \mathbb{R}^{d}}\left\{\langle\theta, \xi\rangle-I_{q}(\xi)\right\}=I_{q}^{*}(\theta)$, the convex conjugate of $I_{q}$ at $\theta$. Similarly, $\Lambda_{a}(\theta)=\log \phi(\theta)=I_{a}^{*}(\theta)$.

For every $N \geq 1, \theta \in \mathbb{R}^{d}$ and $\omega \in \Omega$, define

$$
W_{N}(\theta, \omega):=E_{o}^{\omega}\left[\exp \left\{\left\langle\theta, X_{N}\right\rangle-N \log \phi(\theta)\right\}\right]
$$

Given any $\alpha \in(0,1)$, Jensen's inequality and the bounded convergence theorem imply that

$$
\begin{aligned}
\Lambda_{q}(\theta)-\log \phi(\theta) & =\lim _{N \rightarrow \infty} \frac{1}{N} \log W_{N}(\theta, \cdot)=\mathbb{E}\left[\lim _{N \rightarrow \infty} \frac{1}{N} \log W_{N}(\theta, \cdot)\right] \\
& =\lim _{N \rightarrow \infty} \frac{1}{N} \mathbb{E}\left[\log W_{N}(\theta, \cdot)\right]=\lim _{N \rightarrow \infty} \frac{1}{N \alpha} \mathbb{E}\left[\log W_{N}(\theta, \cdot)^{\alpha}\right] \\
& \leq \limsup _{N \rightarrow \infty} \frac{1}{N \alpha} \log \mathbb{E}\left[W_{N}(\theta, \cdot)^{\alpha}\right] \\
& \leq \lim _{N \rightarrow \infty} \frac{1}{N \alpha} \log \left(\mathbb{E}\left[W_{N}(\theta, \cdot)\right]\right)^{\alpha}=0 .
\end{aligned}
$$

Lemma 2.1. Assume (1.1) and (1.2). Fix any $\alpha \in(0,1)$. If $d=1+1$, then

$$
\limsup _{N \rightarrow \infty} \frac{1}{N} \log \mathbb{E}\left[W_{N}(\theta, \cdot)^{\alpha}\right]<0
$$

whenever $\theta \notin s p\left\{e_{2}\right\}$, the one-dimensional vector space spanned by $e_{2}$.

Lemma 2.2. Assume (1.1) and (1.2). Fix any $\alpha \in(0,1)$. If $d=2+1$, then there exists $a \beta>0$ such that (2.2) holds whenever $\operatorname{dist}\left(\theta, \operatorname{sp}\left\{e_{3}\right\}\right) \in(0, \beta)$.

Remark 2.3. For every $\theta \in \operatorname{sp}\left\{e_{d}\right\},(1.2)$ implies that $W_{N}(\theta, \cdot)=1$ and $\Lambda_{q}(\theta)=$ $\log \phi(\theta)$.

When $d=1+1$, it follows from (2.1) and Lemma 2.1 that $\Lambda_{q}(\cdot)<\log \phi(\cdot)$ on $\left\{\theta \in \mathbb{R}^{2}: \theta \notin \operatorname{sp}\left\{e_{2}\right\}\right\}$. By convex duality, $I_{a}<I_{q}$ on $\left\{\nabla \log \phi(\theta): \theta \notin \operatorname{sp}\left\{e_{2}\right\}\right\}$. It is easy to see that the latter set is equal to $\left((-1,1) \times\left\{e_{2}\right\}\right) \backslash\left\{\xi_{o}\right\}$. In combination with (1.5), this proves Theorem 1.5.

Similarly, when $d=2+1$, Lemma 2.2 implies that $I_{a}<I_{q}$ on $\{\nabla \log \phi(\theta)$ : $\left.\operatorname{dist}\left(\theta, \operatorname{sp}\left\{e_{3}\right\}\right) \in(0, \beta)\right\}$. One can check that this set is of the form $\left(\mathcal{G}_{s t} \times\left\{e_{3}\right\}\right) \backslash\left\{\xi_{o}\right\}$, where $\mathcal{G}_{s t} \subset \mathbb{R}^{2}$ is open and $\mathcal{G}_{s t} \times\left\{e_{3}\right\}$ contains $\xi_{o}$. This proves Theorem 1.6.

The rest of this section is devoted to proving Lemmas 2.1 and 2.2. 
2.2. Decomposing into paths. Assume $d=1+1$ or $2+1$. Let $\mathbb{V}_{d}:=\mathbb{Z}^{d-1} \times\{0\} \subset \mathbb{Z}^{d}$. Fix an $n$ of the form $k^{2}$, with $k$ an integer to be determined later (e.g., for $d=1+1$, this $n$ is chosen so that the conclusion of Lemma 2.4 below holds). When $d=1+1$, let

$$
J_{y}:=\left[\left(y^{\prime}-\frac{1}{2}\right) \sqrt{n},\left(y^{\prime}+\frac{1}{2}\right) \sqrt{n}\right) \times\{0\} \subset \mathbb{R}^{2}
$$

for every $y=\left(y^{\prime}, 0\right) \in \mathbb{V}_{2}$. Similarly, when $d=2+1$, let

$$
J_{y}:=\left[\left(y^{\prime}-\frac{1}{2}\right) \sqrt{n},\left(y^{\prime}+\frac{1}{2}\right) \sqrt{n}\right) \times\left[\left(y^{\prime \prime}-\frac{1}{2}\right) \sqrt{n},\left(y^{\prime \prime}+\frac{1}{2}\right) \sqrt{n}\right) \times\{0\} \subset \mathbb{R}^{3}
$$

for every $y=\left(y^{\prime}, y^{\prime \prime}, 0\right) \in \mathbb{V}_{3}$.

Take $N=n m$ for some $m \geq 1$. For every $\theta \in \mathbb{R}^{d}, \omega \in \Omega$ and $Y=\left(y_{1}, \ldots, y_{m}\right) \in$ $\left(\mathbb{V}_{d}\right)^{m}$, define

$$
\begin{aligned}
\bar{W}_{N}(\theta, \omega, Y):= & E_{o}^{\omega}\left[\exp \left\{\left\langle\theta, X_{N}\right\rangle-N \log \phi(\theta)\right\}, X_{j n}\right. \\
& \left.-\lfloor j n \xi(\theta)\rfloor \in J_{y_{j}} \text { for every } j \leq m\right],
\end{aligned}
$$

where $\xi(\theta)=\nabla \log \phi(\theta)$. (For $u \in \mathbb{R}^{d},\lfloor u\rfloor$ denotes the closest element of $\mathbb{Z}^{d}$ to $u$. If there is more than one closest element, then take the one whose index is the smallest with respect to the lexicographic order.) Note that $\left\langle\xi(\theta), e_{d}\right\rangle=1$ because $\left\langle z, e_{d}\right\rangle=1$ for every $z \in \mathcal{R}_{s t}$.

Since $\mathbb{V}_{d}$ is contained in the disjoint union $\cup_{y \in \mathbb{V}_{d}} J_{y}$, we see that $W_{N}(\theta, \omega)=$ $\sum_{Y} \bar{W}_{N}(\theta, \omega, Y)$. Hence, $W_{N}(\theta, \omega)^{\alpha} \leq \sum_{Y} \bar{W}_{N}(\theta, \omega, Y)^{\alpha}$ by subadditivity, and

$$
\mathbb{E}\left[W_{N}(\theta, \cdot)^{\alpha}\right] \leq \sum_{Y} \mathbb{E}\left[\bar{W}_{N}(\theta, \cdot, Y)^{\alpha}\right]
$$

In the rest of this section, we will treat the cases $d=1+1$ and $d=2+1$ separately.

2.3. Tilting along a path $(d=1+1)$. Our aim is to prove Lemma 2.1 which states that $\mathbb{E}\left[W_{N}(\theta, \cdot)^{\alpha}\right]$ decays exponentially in $N$. Let us say a few words about our strategy. For any function $g(\theta, \cdot)$ on $\Omega$,

$$
\begin{aligned}
\mathbb{E}\left[W_{N}(\theta, \cdot)^{\alpha}\right] & =\mathbb{E}\left[\left(W_{N}(\theta, \cdot) g(\theta, \cdot)\right)^{\alpha} g(\theta, \cdot)^{-\alpha}\right] \\
& \leq \mathbb{E}\left[W_{N}(\theta, \cdot) g(\theta, \cdot)\right]^{\alpha} \mathbb{E}\left[g(\theta, \cdot)^{-\frac{\alpha}{1-\alpha}}\right]^{1-\alpha}
\end{aligned}
$$

by Hölder's inequality. For every $i \geq 1, E_{X_{i}}^{\omega}\left[\exp \left\{\left\langle\theta, X_{i+1}-X_{i}\right\rangle-\log \phi(\theta)\right\}\right]$ and $\left\langle\theta, v\left(T_{X_{i}} \omega\right)-\xi_{o}\right\rangle$ are correlated, cf. (2.23), where $v(\cdot)$ denotes the random drift vector. We could try to exploit this fact by tilting the environment at the points on the path in a clever way, e.g., by choosing a $g(\theta, \cdot)$ that penalizes the environments for which $\frac{1}{N} \sum_{i=1}^{N}\left\langle\theta, v\left(T_{X_{i}} \omega\right)-\xi_{o}\right\rangle$ deviates from zero. This way, we could make the first expectation in (2.6) small. However, there is a problem: we do not know where the path is, and if we naively tilt the environment everywhere, then the second expectation in (2.6) might become too large. Fortunately, it is possible to resolve this issue by first decomposing $\mathbb{E}\left[W_{N}(\theta, \cdot)^{\alpha}\right]$ as in $(2.5)$ (so that we know roughly where the path is), and then tilting the environment on a tube which contains most of the path with a high probability. 
Given $m \geq 1, \theta \notin s p\left\{e_{2}\right\}, C_{1} \geq 1$ and $Y=\left(y_{1}, \ldots, y_{m}\right) \in\left(\mathbb{V}_{2}\right)^{m}$, let

$$
B_{j}:=\left\{(s, i) \in \mathbb{Z}^{2}:(j-1) n \leq i<j n,\left|(s, i)-\lfloor i \xi(\theta)\rfloor-\sqrt{n} y_{j-1}\right| \leq C_{1} \sqrt{n}\right\}
$$

for every $j \in\{1, \ldots, m\}$. Here, $y_{o}=(0,0)$. Recall that $n=k^{2}$ for some integer $k$.

Fix a large $K$ and a small $\delta_{n}$, both to be determined later (depending on the choice of $\alpha$, see (2.12), (2.13) and Lemma 2.4). Define $f_{K}(u):=-K \mathbb{1}_{u \geq \mathrm{e}^{K^{2}}}$ and

$$
g(\theta, \omega, Y):=\exp \sum_{j=1}^{m} f_{K}\left(\delta_{n} D\left(B_{j}\right)\right)>0,
$$

where

$$
D\left(B_{j}\right):=\sum_{(s, i) \in B_{j}} a(\theta,(s, i)) \quad \text { for every } j \in\{1, \ldots, m\},
$$

and $a(\theta, x):=\left\langle\theta, v\left(T_{x} \omega\right)-\xi_{o}\right\rangle$ for every $x \in \mathbb{Z}^{2}$, cf. (1.4). Note that $\mathbb{E}[a(\theta, x)]=0$.

As before, take $N=n m$. By Hölder's inequality,

$$
\begin{aligned}
\mathbb{E}\left[\bar{W}_{N}(\theta, \cdot, Y)^{\alpha}\right] & =\mathbb{E}\left[\left(\bar{W}_{N}(\theta, \cdot Y) g(\theta, \cdot, Y)\right)^{\alpha} g(\theta, \cdot, Y)^{-\alpha}\right] \\
& \leq \mathbb{E}\left[\bar{W}_{N}(\theta, \cdot, Y) g(\theta, \cdot, Y)\right]^{\alpha} \mathbb{E}\left[g(\theta, \cdot, Y)^{-\frac{\alpha}{1-\alpha}}\right]^{1-\alpha} .
\end{aligned}
$$

Let us control the second term in (2.10). $B_{j}$ 's are pairwise disjoint and they each have $n\left(2 C_{1} \sqrt{n}+1\right)$ elements. Since the environment is i.i.d.,

$$
\begin{aligned}
\mathbb{E}\left[g(\theta, \cdot, Y)^{-\frac{\alpha}{1-\alpha}}\right] & =\mathbb{E}\left[\exp \left(-\frac{\alpha}{1-\alpha} \sum_{j=1}^{m} f_{K}\left(\delta_{n} D\left(B_{j}\right)\right)\right)\right] \\
& =\prod_{j=1}^{m} \mathbb{E}\left[\exp \left(-\frac{\alpha}{1-\alpha} f_{K}\left(\delta_{n} D\left(B_{j}\right)\right)\right)\right] \\
& =\mathbb{E}\left[\exp \left(-\frac{\alpha}{1-\alpha} f_{K}\left(\delta_{n} D\left(B_{1}\right)\right)\right)\right]^{m} \\
& \leq\left(1+\mathrm{e}^{\frac{\alpha}{1-\alpha} K} \mathbb{P}\left(\delta_{n} D\left(B_{1}\right) \geq \mathrm{e}^{K^{2}}\right)\right)^{m} .
\end{aligned}
$$

Note that, by Chebyshev's inequality,

$$
\begin{aligned}
\mathbb{P}\left(\delta_{n} D\left(B_{1}\right) \geq \mathrm{e}^{K^{2}}\right) & \leq \mathrm{e}^{-2 K^{2}} \delta_{n}^{2} \mathbb{E}\left[D\left(B_{1}\right)^{2}\right]=\mathrm{e}^{-2 K^{2}} \delta_{n}^{2} \mathbb{E}\left[\sum_{(s, i) \in B_{1}} a(\theta,(s, i))^{2}\right] \\
& =\mathrm{e}^{-2 K^{2}} \delta_{n}^{2} n\left(2 C_{1} \sqrt{n}+1\right) \mathbb{E}\left[a(\theta,(0,0))^{2}\right] \\
& \leq \mathrm{e}^{-2 K^{2}} \delta_{n}^{2} 3 C_{1} n^{3 / 2} \mathbb{E}\left[a(\theta,(0,0))^{2}\right]
\end{aligned}
$$

since, by the i.i.d. assumption on the environment, only the diagonal terms survive. Take

$$
\delta_{n}=C_{1}^{-1 / 2} n^{-3 / 4},
$$


where $C_{1}$ is still to be defined (and will be chosen as in Lemma 2.4). Then, the RHS of (2.11) is bounded from above by

$$
\left(1+3 \mathbb{E}\left[a(\theta,(0,0))^{2}\right] \mathrm{e}^{\frac{\alpha}{1-\alpha} K-2 K^{2}}\right)^{m} \leq\left(1+12 \mathrm{e}^{\frac{\alpha}{1-\alpha} K-2 K^{2}}\right)^{m} \leq 2^{m}
$$

as soon as

$$
12 \mathrm{e}^{\frac{\alpha}{1-\alpha} K-2 K^{2}} \leq 1
$$

Recalling (2.5) and (2.10), we see that

$$
\mathbb{E}\left[W_{N}(\theta, \cdot)^{\alpha}\right] \leq 2^{m} \sum_{Y} \mathbb{E}\left[\bar{W}_{N}(\theta, \cdot, Y) g(\theta, \cdot, Y)\right]^{\alpha} .
$$

2.4. Estimating the expectation under the tilt $(d=1+1)$. For every $m \geq 1, \theta \notin s p\left\{e_{2}\right\}$, $\omega \in \Omega$ and $Y \in\left(\mathbb{V}_{2}\right)^{m}$, let $N=n m$ as before. By the Markov property,

$$
\begin{aligned}
& \bar{W}_{N}(\theta, \omega, Y)=\sum_{x_{1}, \ldots, x_{m} \in \mathbb{Z}^{2}} E_{o}^{\omega}\left[\exp \left\{\left\langle\theta, X_{N}\right\rangle-N \log \phi(\theta)\right\}, X_{j n}-\lfloor j n \xi(\theta)\rfloor\right. \\
& \left.=x_{j} \in J_{y_{j}} \forall j \leq m\right] \\
& =\sum_{x_{1}, \ldots, x_{m} \in \mathbb{Z}^{2}} E_{o}^{\omega}\left[\exp \left\{\left\langle\theta, X_{n}\right\rangle-n \log \phi(\theta)\right\}, X_{n}-\lfloor n \xi(\theta)\rfloor=x_{1} \in J_{y_{1}}\right] \\
& \times E_{x_{1}+\lfloor n \xi(\theta)\rfloor}^{\omega}\left[\exp \left\{\left\langle\theta, X_{n}-\left(x_{1}+\lfloor n \xi(\theta)\rfloor\right)\right\rangle-n \log \phi(\theta)\right\},\right. \\
& \left.X_{n}-\lfloor 2 n \xi(\theta)\rfloor=x_{2} \in J_{y_{2}}\right] \\
& \times \ldots \\
& =\sum_{x_{1}, \ldots, x_{m} \in \mathbb{Z}^{2}} E_{o}^{\omega}\left[\exp \left\{\left\langle\theta, X_{n}\right\rangle-n \log \phi(\theta)\right\}, X_{n}-\lfloor n \xi(\theta)\rfloor=x_{1} \in J_{y_{1}}\right] \\
& \times E_{x_{1}-\sqrt{n} y_{1}}^{T_{\lfloor n \xi(\theta)\rfloor \sqrt{n} y_{1}} \omega}\left[\exp \left\{\left\langle\theta, X_{n}-\left(x_{1}-\sqrt{n} y_{1}\right)\right\rangle-n \log \phi(\theta)\right\},\right. \\
& \left.X_{n}-\lfloor n \xi(\theta)\rfloor=x_{2}-\sqrt{n} y_{1} \in J_{y_{2}}-\sqrt{n} y_{1}\right] \\
& \times \cdots \text {. }
\end{aligned}
$$

Recall (2.8) and (2.9). It follows from the i.i.d. environment assumption that $\mathbb{E}\left[\bar{W}_{N}(\theta, \cdot, Y) g(\theta, \cdot, Y)\right]$

$$
\begin{aligned}
= & \sum_{x_{1}, \ldots, x_{m}} \mathbb{E}\left[E_{o}^{\omega}\left[\exp \left\{\left\langle\theta, X_{n}\right\rangle-n \log \phi(\theta)+f_{K}\left(\delta_{n} D\left(B_{1}\right)\right)\right\}, X_{n}-\lfloor n \xi(\theta)\rfloor=x_{1} \in J_{y_{1}}\right]\right. \\
& \times E_{x_{1}-\sqrt{n} y_{1}}^{T_{\lfloor n \xi(\theta)\rfloor+\sqrt{n} y_{1}} \omega}\left[\exp \left\{\left\langle\theta, X_{n}-\left(x_{1}-\sqrt{n} y_{1}\right)\right\rangle-n \log \phi(\theta)+f_{K}\left(\delta_{n} D\left(B_{1}\right)\right)\right\},\right. \\
& \left.\times X_{n}-\lfloor n \xi(\theta)\rfloor=x_{2}-\sqrt{n} y_{1} \in J_{y_{2}}-\sqrt{n} y_{1}\right] \\
= & \sum_{x_{1}, \ldots, x_{m}} E_{o}\left[\exp \left\{\left\langle\theta, X_{n}\right\rangle-n \log \phi(\theta)+f_{K}\left(\delta_{n} D\left(B_{1}\right)\right)\right\}, X_{n}-\lfloor n \xi(\theta)\rfloor=x_{1} \in J_{y_{1}}\right] \\
& \times E_{x_{1}-\sqrt{n} y_{1}}\left[\exp \left\{\left\langle\theta, X_{n}-\left(x_{1}-\sqrt{n} y_{1}\right)\right\rangle-n \log \phi(\theta)+f_{K}\left(\delta_{n} D\left(B_{1}\right)\right)\right\},\right. \\
\quad & \left.X_{n}-\lfloor n \xi(\theta)\rfloor=x_{2}-\sqrt{n} y_{1} \in J_{y_{2}}-\sqrt{n} y_{1}\right]
\end{aligned}
$$




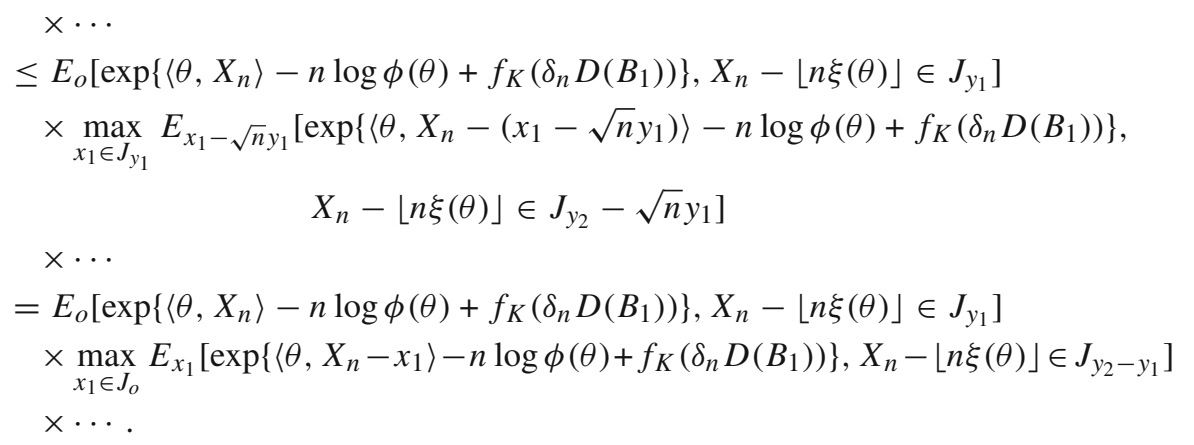

Plugging this in (2.14), we conclude that

$$
\begin{aligned}
\mathbb{E} & {\left[W_{N}(\theta, \cdot)^{\alpha}\right] } \\
& \leq\left(2 \sum_{y \in \mathbb{V}_{2}} \max _{x \in J_{o}} E_{x}\left[\exp \left\{\left\langle\theta, X_{n}-x\right\rangle-n \log \phi(\theta)+f_{K}\left(\delta_{n} D\left(B_{1}\right)\right)\right\}, X_{n}-\lfloor n \xi(\theta)\rfloor \in J_{y}\right]^{\alpha}\right)^{m} .
\end{aligned}
$$

The RHS of this inequality decays exponentially in $m$ if the term in the parentheses is strictly less than 1 . Since $N=n m$ and $n$ was fixed, this proves Lemma 2.1 (and hence Theorem 1.5), provided that we have

Lemma 2.4. Assume (1.1) and (1.2). If $d=1+1, \alpha \in(0,1), \theta \notin \operatorname{sp}\left\{e_{2}\right\}$ and $\delta_{n}=$ $C_{1}^{-1 / 2} n^{-3 / 4}$, then

$$
\sum_{y \in \mathbb{V}_{2}} \max _{x \in J_{o}} E_{x}\left[\exp \left\{\left\langle\theta, X_{n}-x\right\rangle-n \log \phi(\theta)+f_{K}\left(\delta_{n} D\left(B_{1}\right)\right)\right\}, X_{n}-\lfloor n \xi(\theta)\rfloor \in J_{y}\right]^{\alpha}<1 / 2
$$

whenever $n, K$ and $C_{1}$ are sufficiently large.

(The proof is valid with the constant $1 / 2$ replaced by any arbitrarily small positive number.)

\subsection{Finishing the proof of Theorem 1.5. It remains to give the}

Proof of Lemma 2.4. We write the sum in (2.15) as

$$
\left.\sum_{y \in \mathbb{V}_{2}} \max _{x \in J_{o}} E_{x}[\cdots]^{\alpha}=\sum_{\substack{y \in \mathbb{V}_{2}: \\|y|>R}} \max _{x \in J_{o}} E_{x}[\cdots]^{\alpha}+\sum_{\substack{y \in \mathbb{V}_{2}: \\|y| \leq R}} \max _{\substack{x \in J_{o} \\ E_{x}}} E_{x} \cdots\right]^{\alpha}
$$

with some large constant $R$, to be determined. Since $f_{K}(u)=-K \mathbb{1}_{u \geq \mathrm{e}^{K^{2}}} \leq 0$, the first sum on the RHS of (2.16) is bounded from above by

$$
\begin{gathered}
\sum_{\substack{y \in \mathbb{V}_{2}: \\
|y|>R}} \max _{\substack{x \in J_{o} \\
E_{x}}}\left[\exp \left\{\left\langle\theta, X_{n}-x\right\rangle-n \log \phi(\theta)\right\},\left|X_{n}-\lfloor n \xi(\theta)\rfloor-\sqrt{n} y\right| \leq \frac{\sqrt{n}}{2}\right]^{\alpha} \\
\quad \leq \sum_{\substack{y \in \mathbb{V}_{2}: \\
|y|>R}} E_{o}\left[\exp \left\{\left\langle\theta, X_{n}\right\rangle-n \log \phi(\theta)\right\},\left|\frac{X_{n}-\lfloor n \xi(\theta)\rfloor}{\sqrt{n}}-y\right| \leq 1\right]^{\alpha} \cdot
\end{gathered}
$$


Consider a tilted space-time walk on $\mathbb{Z}^{2}$ (in a deterministic environment) with transition probabilities $q^{\theta}(z):=q(z) \exp \{\langle\theta, z\rangle-\log \phi(\theta)\}$ for $z \in \mathcal{R}_{s t}$. Let $\hat{P}_{o}^{\theta}$ denote the probability measure it induces on paths. Note that the LLN velocity under $\hat{P}_{o}^{\theta}$ is

$$
\sum_{z \in \mathcal{R}_{s t}} z q(z) \exp \{\langle\theta, z\rangle-\log \phi(\theta)\}=\nabla \log \phi(\theta)=\xi(\theta)
$$

With this notation, (2.17) is equal to

$$
\sum_{\substack{y \in \mathbb{V}_{2}: \\|y|>R}} \hat{P}_{o}^{\theta}\left(\left|\frac{X_{n}-\lfloor n \xi(\theta)\rfloor}{\sqrt{n}}-y\right| \leq 1\right)^{\alpha} \leq \sum_{\substack{y \in \mathbb{V}_{2}: \\|y|>R}} \hat{P}_{o}^{\theta}\left(\left|\frac{X_{n}-\lfloor n \xi(\theta)\rfloor}{\sqrt{n}}\right| \geq|y|-1\right)^{\alpha}
$$

which, by Chebyshev's inequality, can be made arbitrarily small (uniformly in large $n$ ) by choosing $R$ sufficiently large.

The second sum on the RHS of (2.16) is bounded from above by

$$
(2 R+1) \max _{x \in J_{o}} E_{x}\left[\exp \left\{\left\langle\theta, X_{n}-x\right\rangle-n \log \phi(\theta)+f_{K}\left(\delta_{n} D\left(B_{1}\right)\right)\right\}\right]^{\alpha} .
$$

Therefore, to conclude the proof of Lemma 2.4, it suffices to show that

$$
E_{o}\left[\exp \left\{\left\langle\theta, X_{n}\right\rangle-n \log \phi(\theta)+f_{K}\left(\delta_{n} D\left(B_{1}-x\right)\right)\right\}\right] \leq\left(\frac{1}{8 R}\right)^{\alpha^{-1}}
$$

for every $x \in J_{o}$.

Similar to $B_{1}$ defined in (2.7), introduce a new set

$$
\bar{B}_{1}:=\left\{(s, i) \in \mathbb{Z}^{2}: 0 \leq i<n,|(s, i)-\lfloor i \xi(\theta)\rfloor| \leq\left(C_{1}-1 / 2\right) \sqrt{n}\right\} .
$$

Note that $\bar{B}_{1} \subset B_{1}-x$ for every $x \in J_{o}$ since $|x| \leq \sqrt{n} / 2$. We have

$$
\begin{aligned}
E_{o} & {\left[\exp \left\{\left\langle\theta, X_{n}\right\rangle-n \log \phi(\theta)+f_{K}\left(\delta_{n} D\left(B_{1}-x\right)\right)\right\}\right] } \\
= & \mathrm{e}^{-K} E_{o}\left[\exp \left\{\left\langle\theta, X_{n}\right\rangle-n \log \phi(\theta)\right\}, \delta_{n} D\left(B_{1}-x\right) \geq \mathrm{e}^{K^{2}}\right] \\
& +E_{o}\left[\exp \left\{\left\langle\theta, X_{n}\right\rangle-n \log \phi(\theta)\right\},\left\{X_{i}: 0 \leq i<n\right\} \not \subset \bar{B}_{1}, \delta_{n} D\left(B_{1}-x\right)<\mathrm{e}^{K^{2}}\right] \\
& +E_{o}\left[\exp \left\{\left\langle\theta, X_{n}\right\rangle-n \log \phi(\theta)\right\},\left\{X_{i}: 0 \leq i<n\right\} \subset \bar{B}_{1}, \delta_{n} D\left(B_{1}-x\right)<\mathrm{e}^{K^{2}}\right] \\
\leq & \mathrm{e}^{-K}+\hat{P}_{o}^{\theta}\left(\left\{X_{i}: 0 \leq i<n\right\} \not \subset \bar{B}_{1}\right) \\
& +E_{o}\left[\exp \left\{\left\langle\theta, X_{n}\right\rangle-n \log \phi(\theta)\right\},\left\{X_{i}: 0 \leq i<n\right\} \subset \bar{B}_{1}, \delta_{n} D\left(B_{1}-x\right)<\mathrm{e}^{K^{2}}\right] .
\end{aligned}
$$

The first term in (2.19) is small when $K$ is large. Donsker's invariance principle ensures that the second term can be made arbitrarily small (uniformly in $n$ ) by choosing $C_{1}$ sufficiently large. 
Let us focus on the third term in (2.19). For any sequence $\left(A_{n}\right)_{n \geq 1}$ of natural numbers,

$$
\begin{aligned}
& E_{o}\left[\exp \left\{\left\langle\theta, X_{n}\right\rangle-n \log \phi(\theta)\right\},\left\{X_{i}: 0 \leq i<n\right\} \subset \bar{B}_{1}, \delta_{n} D\left(B_{1}-x\right)<\mathrm{e}^{K^{2}}\right] \\
& \leq E_{o}\left[\exp \left\{\left\langle\theta, X_{n}\right\rangle-n \log \phi(\theta)\right\},\left\{X_{i}: 0 \leq i<n\right\} \subset \bar{B}_{1}, \delta_{n} \sum_{\substack{(s, i) \in B_{1}-x \\
(s, i) \neq X_{i}}} a(\theta,(s, i))<-A_{n}\right] \\
& +E_{o}\left[\exp \left\{\left\langle\theta, X_{n}\right\rangle-n \log \phi(\theta)\right\},\left\{X_{i}: 0 \leq i<n\right\} \subset \bar{B}_{1}, \delta_{n} \sum_{i=0}^{n-1} a\left(\theta, X_{i}\right)<\mathrm{e}^{K^{2}}+A_{n}\right] \\
& \leq \sum_{x_{1}, \ldots, x_{n-1}} \mathbb{E}\left[E_{o}^{\omega}\left[\exp \left\{\left\langle\theta, X_{n}\right\rangle-n \log \phi(\theta)\right\}, X_{i}=x_{i} \forall i<n\right],\right. \\
& \left.\delta_{n} \sum_{\substack{(s, i) \in B_{1}-x \\
(s, i) \neq x_{i}}} a(\theta,(s, i))<-A_{n}\right] \\
& +E_{o}\left[\exp \left\{\left\langle\theta, X_{n}\right\rangle-n \log \phi(\theta)\right\}, \delta_{n} \sum_{i=0}^{n-1} a\left(\theta, X_{i}\right)<\mathrm{e}^{K^{2}}+A_{n}\right] \\
& =\sum_{x_{1}, \ldots, x_{n-1}} E_{o}\left[\exp \left\{\left\langle\theta, X_{n}\right\rangle-n \log \phi(\theta)\right\}, X_{i}=x_{i} \forall i<n\right] \\
& \times \mathbb{P}\left(\delta_{n} \sum_{\substack{(s, i) \in B_{1}-x \\
(s, i) \neq x_{i}}} a(\theta,(s, i))<-A_{n}\right) \\
& +E_{o}\left[\exp \left\{\left\langle\theta, X_{n}\right\rangle-n \log \phi(\theta)\right\}, \delta_{n} \sum_{i=0}^{n-1} a\left(\theta, X_{i}\right)<\mathrm{e}^{K^{2}}+A_{n}\right] \\
& \leq \max _{x_{1}, \ldots, x_{n-1}} \mathbb{P}\left(\delta_{n} \sum_{\substack{(s, i) \in B_{1}-x \\
(s, i) \neq x_{i}}} a(\theta,(s, i))<-A_{n}\right) \\
& +E_{o}\left[\exp \left\{\left\langle\theta, X_{n}\right\rangle-n \log \phi(\theta)\right\}, \delta_{n} \sum_{i=0}^{n-1} a\left(\theta, X_{i}\right)<\mathrm{e}^{K^{2}}+A_{n}\right] \\
& \leq A_{n}^{-2} \delta_{n}^{2} 2 C_{1} n^{3 / 2} \mathbb{E}\left[a(\theta,(0,0))^{2}\right] \\
& +E_{o}\left[\exp \left\{\left\langle\theta, X_{n}\right\rangle-n \log \phi(\theta)\right\}, \delta_{n} \sum_{i=0}^{n-1} a\left(\theta, X_{i}\right)<\mathrm{e}^{K^{2}}+A_{n}\right] .
\end{aligned}
$$

Here, (2.20) follows from the independence assumption on the environment, and (2.21) is an application of Chebyshev's inequality. Since $\delta_{n}=C_{1}^{-1 / 2} n^{-3 / 4}$, the first term in (2.21) goes to zero as $n \rightarrow \infty$ if $A_{n} \rightarrow \infty$. 
Choose $A_{n}$ such that $A_{n} \rightarrow \infty$ and $A_{n}=o\left(n^{1 / 4}\right)$ as $n \rightarrow \infty$. For any $\mu \in \mathbb{R}^{+}$, the second term in (2.21) is equal to

$$
\begin{aligned}
& E_{o}\left[\exp \left\{\left\langle\theta, X_{n}\right\rangle-n \log \phi(\theta)\right\}, \delta_{n} \sum_{i=0}^{n-1}\left(a\left(\theta, X_{i}\right)-\mu\right)<\mathrm{e}^{K^{2}}+A_{n}-\mu n \delta_{n}\right] \\
& \leq M_{n} E_{o}\left[\exp \left\{\left\langle\theta, X_{n}\right\rangle-n \log \phi(\theta)\right\} \sum_{i=0}^{n-1}\left(a\left(\theta, X_{i}\right)-\mu\right)^{2}\right] \\
& \quad+M_{n} E_{o}\left[\exp \left\{\left\langle\theta, X_{n}\right\rangle-n \log \phi(\theta)\right\} \sum_{i \neq j}\left(a\left(\theta, X_{i}\right)-\mu\right)\left(a\left(\theta, X_{j}\right)-\mu\right)\right]
\end{aligned}
$$

by Chebyshev's inequality, where $M_{n}=\left(\frac{\delta_{n}}{\mu n \delta_{n}-A_{n}-\mathrm{e}^{K^{2}}}\right)^{2}=O\left(n^{-2}\right)$.

By the FKG inequality (cf. [8]),

$$
\begin{aligned}
& E_{o}\left[\exp \left\{\left\langle\theta, X_{1}\right\rangle-\log \phi(\theta)\right\} a(\theta,(0,0))\right] \\
& \quad=\mathbb{E}\left[E_{o}^{\omega}\left[\exp \left\{\left\langle\theta, X_{1}\right\rangle-\log \phi(\theta)\right\}\right] a(\theta,(0,0))\right] \\
& >\mathbb{E}\left[E_{o}^{\omega}\left[\exp \left\{\left\langle\theta, X_{1}\right\rangle-\log \phi(\theta)\right\}\right]\right] \mathbb{E}[a(\theta,(0,0))]=0,
\end{aligned}
$$

since $E_{o}^{\omega}\left[\exp \left\{\left\langle\theta, X_{1}\right\rangle-\log \phi(\theta)\right\}\right]$ and $a(\theta,(0,0))$ are easily checked to be either both strictly increasing functions (when $\left\langle\theta, e_{1}\right\rangle>0$ ) or both strictly decreasing functions (when $\left.\left\langle\theta, e_{1}\right\rangle<0\right)$ of the random variable $\pi((0,0),(1,1))$. If we choose

$$
\mu=E_{o}\left[\exp \left\{\left\langle\theta, X_{1}\right\rangle-\log \phi(\theta)\right\} a(\theta,(0,0))\right],
$$

then the second term in (2.22) vanishes by the independence assumption on the environment. Finally, observe that the first term in (2.22) is equal to

$$
n M_{n} E_{o}\left[\exp \left\{\left\langle\theta, X_{1}\right\rangle-\log \phi(\theta)\right\}(a(\theta,(0,0))-\mu)^{2}\right]=O\left(n^{-1}\right) .
$$

2.6. Proof of Theorem 1.6. Let us recall a few points regarding the arguments in Subsects. 2.3-2.5. There, since $d=1+1$, the volume of $B_{1}$ (defined in (2.7)) is $O\left(n^{3 / 2}\right)$. The variance of $D\left(B_{1}\right)$ (cf. (2.9)) scales like that volume. We take $\delta_{n}=O\left(n^{-3 / 4}\right)$ so that the variance of $\delta_{n} D\left(B_{1}\right)$ is $O(1)$. With this choice, $n \delta_{n} \rightarrow \infty$ as $n \rightarrow \infty$. As we saw, this fact is crucial in the proof of Theorem 1.5.

In this subsection, we will assume that $d=2+1$. For every $m \geq 1,1 \leq j \leq m$, $\theta \notin \operatorname{sp}\left\{e_{3}\right\}, C_{1} \geq 1$ and $Y=\left(y_{1}, \ldots, y_{m}\right) \in\left(\mathbb{V}_{3}\right)^{m}$, we define

$$
B_{j}:=\left\{(r, k): r \in \mathbb{Z}^{2},(j-1) n \leq k<j n,\left|(r, k)-\lfloor k \xi(\theta)\rfloor-\sqrt{n} y_{j-1}\right| \leq C_{1} \sqrt{n}\right\},
$$

similar to (2.7). Note that the volume of this new set is $O\left(n^{2}\right)$. If we were to define $D\left(B_{1}\right)$ analogously to (2.9), then we would have to take $\delta_{n} \leq O\left(n^{-1}\right)$ in order to make the variance of $\delta_{n} D\left(B_{1}\right)$ not grow with $n$, in which case $n \delta_{n}$ remains bounded. Hence, the proof for $d=1+1$ does not directly carry over to the case $d=2+1$. 
To resolve this issue, following [10], we will modify the proof by redefining $D\left(B_{1}\right)$ and $\delta_{n}$. (We will continue using these names so that we can refer to the parts of Subsects. 2.3-2.5 that carry over word by word.) The modification amounts essentially to using a tilting that is quadratic, instead of linear, in the local drift, as follows.

For every $(r, k)$ and $(s, l)$ with $r, s \in \mathbb{Z}^{2}$ and $k, l \geq 1$, let

$$
V((r, k),(s, l)):=\frac{1}{|k-l|} \mathbb{1}_{\left\{|(s, l)-(r, k)-\lfloor(l-k) \xi(\theta)\rfloor|<C_{2} \sqrt{|k-l|\}}\right.}
$$

if $k \neq l$, and set it to be equal to zero if $k=l$. Here, the constant $C_{2} \geq 1$ will be determined later. Given any $n$ integer and $x_{1}, \ldots, x_{n} \in \mathbb{Z}^{3}$ with $\left\langle x_{k}, e_{3}\right\rangle=k$, it follows easily from (2.26) that

$$
\begin{aligned}
& \text { for any } s \in \mathbb{Z}^{2}, \quad l \in\{1, \ldots, n\}, \quad \sum_{k=1}^{n} V\left(x_{k},(s, l)\right) \leq 2 \log n, \\
& \sum_{k=1}^{n} \sum_{(s, l) \in B_{1}} V\left(x_{k},(s, l)\right) \leq \sum_{\substack{1 \leq k, l \leq n \\
k \neq l}} \frac{1}{|k-l|}\left(2 C_{2} \sqrt{|k-l|}\right)^{2} \leq 4 C_{2}^{2} n^{2}, \\
& \sum_{(s, l) \in B_{1}}\left(\sum_{k=1}^{n} V\left(x_{k},(s, l)\right)\right)^{2}=\left(\max _{\left(s^{\prime}, l^{\prime}\right)} \sum_{k=1}^{n} V\left(x_{k},\left(s^{\prime}, l^{\prime}\right)\right)\right) \sum_{(s, l) \in B_{1}} \sum_{k=1}^{n} V\left(x_{k},(s, l)\right) \\
& \leq(2 \log n)\left(4 C_{2}^{2} n^{2}\right)=8 C_{2}^{2} n^{2} \log n, \quad \text { and } \\
& \sum_{\substack{(r, k) \in B_{1},(s, l) \in B_{1}}} V((r, k),(s, l))^{2} \leq \sum_{k=1}^{n}\left(2 C_{1} \sqrt{n}\right)^{2} \sum_{l=1}^{n} \frac{\mathbb{1}_{\{k \neq l\}}}{|k-l|^{2}}\left(2 C_{2} \sqrt{|k-l|}\right)^{2} \\
& =16 C_{1}^{2} C_{2}^{2} n \sum_{\substack{1 \leq k, l \leq n \\
k \neq l}} \frac{1}{|k-l|} \leq 32 C_{1}^{2} C_{2}^{2} n^{2} \log n .
\end{aligned}
$$

Recall the tilted law $\hat{P}_{o}^{\theta}$ introduced in the proof of Lemma 2.4.

Lemma 2.5. For any $\delta>0$, there exists a $C_{2} \geq 1$ such that $v(n, X):=\sum_{1 \leq i, j \leq n} V\left(X_{i}\right.$, $\left.X_{j}\right)$ satisfies

$$
\hat{P}_{o}^{\theta}(v(n, X)<n \log (n-1) / 2) \leq \delta
$$

for every $n \geq 2$.

Proof. For any realization of $X=\left(X_{i}\right)_{i \geq 1}$,

$$
v(n, X) \leq \sum_{\substack{1 \leq i, j \leq n \\ i \neq j}} \frac{1}{|i-j|}=: H(n)
$$


Observe that

$$
\begin{aligned}
\hat{E}_{o}^{\theta}[v(n, X)] & =\sum_{1 \leq i, j \leq n} \hat{E}_{o}^{\theta}\left[V\left(X_{i}, X_{j}\right)\right] \\
& =\sum_{\substack{1 \leq i, j \leq n \\
i \neq j}} \frac{1}{|i-j|} \hat{P}_{o}^{\theta}\left(\left|X_{i}-X_{j}-\lfloor(i-j) \xi(\theta)\rfloor\right|<C_{2} \sqrt{|i-j|}\right) .
\end{aligned}
$$

When $C_{2}$ is sufficiently large, the CLT implies that

$$
\hat{P}_{o}^{\theta}\left(\left|X_{i}-X_{j}-\lfloor(i-j) \xi(\theta)\rfloor\right|<C_{2} \sqrt{|i-j|}\right) \geq(1-\delta / 2)
$$

for any $i \neq j$. Therefore, $\hat{E}_{o}^{\theta}[v(n, X)] \geq(1-\delta / 2) H(n)$. Applying Markov's inequality, we see that

$$
\hat{P}_{o}^{\theta}(v(n, X)<H(n) / 2)=\hat{P}_{o}^{\theta}(H(n)-v(n, X)>H(n) / 2) \leq \delta .
$$

This implies the desired result since $H(n) \geq n \log (n-1)$.

For any $\theta \in \mathbb{R}^{3}$ and $x \in \mathbb{Z}^{3}$, define $a(\theta, x):=\left\langle\theta, v\left(T_{x} \omega\right)-\xi_{o}\right\rangle$ as before, where $v(\omega)=\sum_{z \in \mathcal{R}} \pi(0, z) z$.

Lemma 2.6. There exists a $\beta>0$ such that

$$
\mu:=E_{o}\left[\exp \left\{\left\langle\theta, X_{1}\right\rangle-\log \phi(\theta)\right\} a(\theta,(0,0,0))\right]>0
$$

whenever $\operatorname{dist}\left(\theta, \operatorname{sp}\left\{e_{3}\right\}\right) \in(0, \beta)$.

Proof. For every $\theta \notin s p\left\{e_{3}\right\}$, let

$$
\begin{aligned}
& F(\theta):=\mathbb{E}\left\{E_{o}^{\omega}\left[\mathrm{e}^{\left\langle\theta, X_{1}\right\rangle}\right] E_{o}^{\omega}\left[\left\langle\theta, X_{1}\right\rangle\right]\right\} \text { and } \\
& G(\theta):=E_{o}\left[\mathrm{e}^{\left\langle\theta, X_{1}\right\rangle}\right] E_{o}\left[\left\langle\theta, X_{1}\right\rangle\right]=\phi(\theta)\left\langle\theta, \xi_{o}\right\rangle .
\end{aligned}
$$

Our aim is to show that $F(\theta)>G(\theta)$.

Write $\theta=c e_{3}+\theta^{\prime}$ for some $c \in \mathbb{R}$ and $\theta^{\prime} \in \mathbb{R}^{3}$ such that $\left\langle\theta^{\prime}, e_{3}\right\rangle=0$. Then, $F(\theta)=\mathrm{e}^{c} F\left(\theta^{\prime}\right)+c \mathrm{e}^{c} \phi\left(\theta^{\prime}\right)$ and $G(\theta)=\mathrm{e}^{c} G\left(\theta^{\prime}\right)+c \mathrm{e}^{c} \phi\left(\theta^{\prime}\right)$. Therefore, it suffices to show that $F\left(\theta^{\prime}\right)>G\left(\theta^{\prime}\right)$.

Clearly, we have

$$
\left.\nabla F(\theta)\right|_{\theta=0}=\left.\nabla G(\theta)\right|_{\theta=0}=E_{o}\left[X_{1}\right]=\xi_{o} .
$$

Also, for any $u, u^{\prime} \in \mathbb{R}^{3}$, with $D^{2} F$ denoting the Hessian of $F$,

$$
\left.\left\langle u, D^{2} F(\theta) u^{\prime}\right\rangle\right|_{\theta=0}=2 \mathbb{E}\left\{E_{o}^{\omega}\left[\left\langle X_{1}, u\right\rangle\right] E_{o}^{\omega}\left[\left\langle X_{1}, u^{\prime}\right\rangle\right]\right\}
$$

and

$$
\left.\left\langle u, D^{2} G(\theta) u^{\prime}\right\rangle\right|_{\theta=0}=2 E_{o}\left[\left\langle X_{1}, u\right\rangle\right] E_{o}\left[\left\langle X_{1}, u^{\prime}\right\rangle\right]=2\left\langle\xi_{o}, u\right\rangle\left\langle\xi_{o}, u^{\prime}\right\rangle .
$$

By Schwarz' inequality (which is strict since the walk is uniformly elliptic in the directions other than $e_{3}$ ),

$$
\inf _{\substack{|u|=1 \\\left\langle u, e_{3}\right\rangle=0}}\left(\left.\left\langle u, D^{2} F(\theta) u\right\rangle\right|_{\theta=0}-\left.\left\langle u, D^{2} G(\theta) u\right\rangle\right|_{\theta=0}\right)>0 .
$$

Finally, Taylor's theorem implies the existence of a $\beta>0$ such that $F\left(\theta^{\prime}\right)>G\left(\theta^{\prime}\right)$ whenever $\left|\theta^{\prime}\right| \in(0, \beta)$. 
Now, we are ready to give the new definition of $D\left(B_{1}\right)$ which is suitable for $d=2+1$. For any $\theta \in \mathbb{R}^{3}$ such that $\operatorname{dist}\left(\theta, \operatorname{sp}\left\{e_{3}\right\}\right) \in(0, \beta)$ (with $\beta$ as in Lemma 2.6), let

$$
D\left(B_{1}\right):=\sum_{\substack{(r, k) \in B_{1},(s, l) \in B_{1}}} V((r, k),(s, l)) a(\theta,(r, k)) a(\theta,(s, l)) .
$$

Note that $V((\cdot, k),(\cdot, k))=0$ for every $1 \leq k \leq n$. Since $\mathbb{E}[a(\theta, 0)]=0$, it follows from the independence of the environment that $\mathbb{E}\left[D\left(B_{1}\right)\right]=0$. Also, $\mathbb{E}\left[D\left(B_{1}\right)^{2}\right] \leq$ $1024|\theta|^{4} C_{1}^{2} C_{2}^{2} n^{2} \log n$ by (2.28) and the fact that $|a(\theta, 0)| \leq 2|\theta|$.

If we choose

$$
\delta_{n}:=n^{-1}(\log n)^{-1 / 2},
$$

then the variance of $\delta_{n} D\left(B_{1}\right)$ is $O(1)$. Once we have this fact, the arguments in Subsects. 2.3-2.5 carry over until (2.18). So, it suffices to show that $E_{o}\left[\exp \left\{\left\langle\theta, X_{n}\right\rangle-\right.\right.$ $\left.n \log \phi(\theta)\}, \delta_{n} D\left(B_{1}-x\right)<\mathrm{e}^{K^{2}}\right]$ is small for all $x \in J_{o}$ when $n$ and $K$ are large. In the estimate below, we will (WLOG) take $x=0$.

Let $\gamma=1 / 2$, and observe that

$$
\begin{aligned}
& E_{o}\left[\exp \left\{\left\langle\theta, X_{n}\right\rangle-n \log \phi(\theta)\right\}, \delta_{n} D\left(B_{1}\right)<\mathrm{e}^{K^{2}}\right] \\
& \leq E_{o}\left[\exp \left\{\left\langle\theta, X_{n}\right\rangle-n \log \phi(\theta)\right\}, v(n, X) \geq \gamma n \log (n-1), \delta_{n} D\left(B_{1}\right)<\mathrm{e}^{K^{2}}\right] \\
&+E_{o}\left[\exp \left\{\left\langle\theta, X_{n}\right\rangle-n \log \phi(\theta)\right\}, v(n, X)<\gamma n \log (n-1)\right] \\
&= E_{o}\left[\exp \left\{\left\langle\theta, X_{n}\right\rangle-n \log \phi(\theta)\right\}, v(n, X) \geq \gamma n \log (n-1),\right. \\
&\left.\delta_{n}\left(D\left(B_{1}\right)-\mu^{2} v(n, X)\right)<\mathrm{e}^{K^{2}}-\mu^{2} \delta_{n} v(n, X)\right]+\hat{P}_{o}^{\theta}(v(n, X)<\gamma n \log (n-1)) \\
& \leq M_{n} E_{o}\left[\exp \left\{\left\langle\theta, X_{n}\right\rangle-n \log \phi(\theta)\right\}\left(D\left(B_{1}\right)-\mu^{2} v(n, X)\right)^{2}, v(n, X) \geq \gamma n \log (n-1)\right] \\
&+\hat{P}_{o}^{\theta}(v(n, X)<\gamma n \log (n-1)) \\
& \leq M_{n} E_{o}\left[\exp \left\{\left\langle\theta, X_{n}\right\rangle-n \log \phi(\theta)\right\}\left(D\left(B_{1}\right)-\mu^{2} v(n, X)\right)^{2}\right] \\
&+\hat{P}_{o}^{\theta}(v(n, X)<\gamma n \log (n-1)) .
\end{aligned}
$$

Here, (2.30) follows from the elementary inequality $\mathbb{1}_{a<b} \leq a^{2} / b^{2}$ with $a=\delta_{n}\left(D\left(B_{1}\right)-\right.$ $\left.\mu^{2} v(n, X)\right)$ and $b=\mathrm{e}^{K^{2}}-\mu^{2} \delta_{n} v(n, X)<0$, and

$$
M_{n}=\left(\frac{\delta_{n}}{\mu^{2} \delta_{n} \gamma n \log (n-1)-\mathrm{e}^{K^{2}}}\right)^{2} .
$$

Choose $C_{2}$ sufficiently large so that the second term in (2.31) is small for all $n \geq 2$ by Lemma 2.5.

It remains to control the first term in (2.31). Note that

$$
\begin{aligned}
D\left(B_{1}\right)-\mu^{2} v(n, X) \\
=2 \mu \sum_{k=1}^{n} \sum_{(s, l) \in B_{1}} V\left(X_{k},(s, l)\right)\left(a(\theta,(s, l))-\mu \mathbb{1}_{\left\{X_{l}=(s, l)\right\}}\right) \\
\quad+\sum_{\substack{(r, k) \in B_{1},(s, l) \in B_{1}}} V((r, k),(s, l))\left(a(\theta,(r, k))-\mu \mathbb{1}_{\left\{X_{k}=(r, k)\right\}}\right)\left(a(\theta,(s, l))-\mu \mathbb{1}_{\left\{X_{l}=(s, l)\right\}}\right),
\end{aligned}
$$


and

$$
\begin{aligned}
& E_{o}\left[\exp \left\{\left\langle\theta, X_{n}\right\rangle-n \log \phi(\theta)\right\}\left(D\left(B_{1}\right)-\mu^{2} v(n, X)\right)^{2}\right] \\
& \leq 8 \mu^{2} E_{o}\left[\exp \{\cdots\}\left(\sum_{k=1}^{n} \sum_{(s, l) \in B_{1}} V\left(X_{k},(s, l)\right)\left(a(\theta,(s, l))-\mu \mathbb{1}_{\left\{X_{l}=(s, l)\right\}}\right)\right)^{2}\right] \\
& +2 E_{o}\left[\operatorname { e x p } \{ \cdots \} \left(\sum_{\substack{(r, k) \in B_{1},(s, l) \in B_{1}}} V((r, k),(s, l))\left(a(\theta,(r, k))-\mu \mathbb{1}_{\left\{X_{k}=(r, k)\right\}}\right)(a(\theta,(s, l))\right.\right. \\
& \left.\left.\left.-\mu \mathbb{1}_{\left\{X_{l}=(s, l)\right\}}\right)\right)^{2}\right] \\
& =: 8 \mu^{2} \mathfrak{E}_{1}+2 \mathfrak{E}_{2}
\end{aligned}
$$

by the inequality $(a+b)^{2} \leq 2\left(a^{2}+b^{2}\right)$.

One should note at this stage that in fact, even though $\mu$ was chosen to equal the mean under the tilted measure of $a(\theta, 0)$, it is not necessarily the case that the mean of $D\left(B_{1}\right)-\mu^{2} v(n, X)$ under that tilted measure vanishes. This makes the control of $\mathfrak{E}_{i}$ somewhat messy, involving a local CLT (Lemma 2.7).

We turn to the details of the computation. $\mathfrak{E}_{1}$ can be written as a double sum over pairs $(s, l),\left(s^{\prime}, l^{\prime}\right) \in B_{1}$. If $(s, l) \neq\left(s^{\prime}, l^{\prime}\right)$, then it is clear from independence that this pair does not contribute to $\mathfrak{E}_{1}$ on the event $\left\{X_{l} \neq(s, l)\right\} \cup\left\{X_{l^{\prime}} \neq\left(s^{\prime}, l^{\prime}\right)\right\}$. Therefore,

$$
\begin{aligned}
\mathfrak{E}_{1}= & E_{o}\left[\exp \{\cdots\} \sum_{(s, l) \in B_{1}}\left(\sum_{k=1}^{n} V\left(X_{k},(s, l)\right)\left(a(\theta,(s, l))-\mu \mathbb{1}_{\left\{X_{l}=(s, l)\right\}}\right)\right)^{2}\right] \\
& +\sum_{\substack{k, k^{\prime}, l, l^{\prime}: \\
l \neq l^{\prime}}} E_{o}\left[\exp \left\{\left\langle\theta, X_{n}\right\rangle-n \log \phi(\theta)\right\} V\left(X_{k}, X_{l}\right) V\left(X_{k^{\prime}}, X_{l^{\prime}}\right)\right. \\
& \left.\times\left(a\left(\theta, X_{l}\right)-\mu\right)\left(a\left(\theta, X_{l^{\prime}}\right)-\mu\right)\right] \\
= & E_{o}\left[\exp \{\cdots\} \sum_{(s, l) \in B_{1}}\left(\sum_{k=1}^{n} V\left(X_{k},(s, l)\right)\left(a(\theta,(s, l))-\mu \mathbb{1}_{\left\{X_{l}=(s, l)\right\}}\right)\right)^{2}\right] \\
& +\sum_{\substack{k, k^{\prime}, l, l^{\prime}: \\
l \neq l^{\prime}}} \mathfrak{E}_{1}\left(k, k^{\prime}, l, l^{\prime}\right) \\
\leq & (2|\theta|+\mu)^{2} E_{o}\left[\exp \{\cdots\} \sum_{\substack{(s, l) \in B_{1} \\
k=1}}\left(\sum_{k=1}^{n} V\left(X_{k},(s, l)\right)\right)^{2}\right]+\sum_{\substack{k, k^{\prime}, l, l^{\prime}: \\
l \neq l^{\prime}}} \mathfrak{E}_{1}\left(k, k^{\prime}, l, l^{\prime}\right) \\
\leq & (2|\theta|+\mu)^{2} 8 C_{2}^{2} n^{2} \log n+\sum_{\substack{k, k^{\prime}, l, l^{\prime}: \\
l \neq l^{\prime}}} \mathfrak{E}_{1}\left(k, k^{\prime}, l, l^{\prime}\right)
\end{aligned}
$$

by (2.27) and the fact that $|a(\theta, \cdot)| \leq 2|\theta|$. 
If $l^{\prime}>\max \left(k, k^{\prime}, l\right)$, then $\mathfrak{E}_{1}\left(k, k^{\prime}, l, l^{\prime}\right)$ is equal to zero since we can condition on the path up to $l^{\prime}$ and use the fact that, for any $\left(x_{i}\right)_{1}^{l^{\prime}}$,

$$
E_{o}\left[\exp \left\{\left\langle\theta, X_{n}-X_{l^{\prime}}\right\rangle-\left(n-l^{\prime}\right) \log \phi(\theta)\right\}\left(a\left(\theta, X_{l^{\prime}}\right)-\mu\right) \mid\left(X_{i}\right)_{1}^{l^{\prime}}=\left(x_{i}\right)_{1}^{l^{\prime}}\right]=0
$$

by the definition of $\mu$, cf. Lemma 2.6.

If $l<l^{\prime}<k^{\prime}<k$, then $V\left(X_{k}, X_{l}\right)$ and $V\left(X_{k^{\prime}}, X_{l^{\prime}}\right)$ create a slight complication since $X_{k}$ and $X_{k^{\prime}}$ are not independent of $X_{l^{\prime}+1}-X_{l^{\prime}}$. Indeed,

$$
\begin{aligned}
& \mathfrak{E}_{1}\left(k, k^{\prime}, l, l^{\prime}\right) \\
& =\sum_{\substack{x_{1}, \ldots, x_{l^{\prime}} \\
z \in \mathcal{R}}} E_{o}\left[\exp \{\cdots\}\left(a\left(\theta, X_{l}\right)-\mu\right)\left(a\left(\theta, X_{l^{\prime}}\right)-\mu\right),\left(X_{i}\right)_{1}^{l^{\prime}}=\left(x_{i}\right)_{1}^{l^{\prime}}, X_{l^{\prime}+1}-X_{l^{\prime}}=z\right] \\
& \quad \times \hat{E}_{o}^{\theta}\left[V\left(X_{k}, x_{l}\right) V\left(X_{k^{\prime}}, x_{l^{\prime}}\right) \mid X_{l^{\prime}+1}=x_{l^{\prime}}+z\right],
\end{aligned}
$$

and the latter expectation depends on $z$. (If it were independent of $z$, we could simply take the sum over $z \in \mathcal{R}$ and conclude that $\mathfrak{E}_{1}\left(k, k^{\prime}, l, l^{\prime}\right)=0$.) However, for any $z$, $z^{\prime} \in \mathcal{R}$

$$
\begin{aligned}
& \left|\hat{E}_{o}^{\theta}\left[V\left(X_{k}, x_{l}\right) V\left(X_{k^{\prime}}, x_{l^{\prime}}\right) \mid X_{l^{\prime}+1}=x_{l^{\prime}}+z\right]-\hat{E}_{o}^{\theta}\left[V\left(X_{k}, x_{l}\right) V\left(X_{k^{\prime}}, x_{l^{\prime}}\right) \mid X_{l^{\prime}+1}=x_{l^{\prime}}+z^{\prime}\right]\right| \\
& \leq \sum_{\substack{x_{k^{\prime}}: \\
V\left(x_{k^{\prime}}, x_{l^{\prime}}\right)>0}}\left(k^{\prime}-l^{\prime}\right)^{-1}\left|\hat{P}_{o}^{\theta}\left(X_{k^{\prime}}=x_{k^{\prime}} \mid X_{l^{\prime}+1}=x_{l^{\prime}}+z\right)-\hat{P}_{o}^{\theta}\left(X_{k^{\prime}}=x_{k^{\prime}} \mid X_{l^{\prime}+1}=x_{l^{\prime}}+z^{\prime}\right)\right| \\
& \quad \times \hat{E}_{o}^{\theta}\left[V\left(X_{k}, x_{l}\right) \mid X_{k^{\prime}}=x_{k^{\prime}}\right] \\
& \leq 4 C_{2}^{2}\left(k^{\prime}-l^{\prime}\right)\left(k^{\prime}-l^{\prime}\right)^{-1} O\left(\left(k^{\prime}-l^{\prime}\right)^{-3 / 2}\right)(k-l)^{-1}=O\left((k-l)^{-1}\left(k^{\prime}-l^{\prime}\right)^{-3 / 2}\right)
\end{aligned}
$$

uniformly in $\left(x_{i}\right)_{1}^{l^{\prime}}$, cf. Lemma 2.7 (given below). Hence,

$$
\sum_{l<l^{\prime}<k^{\prime}<k} \mathfrak{E}_{1}\left(k, k^{\prime}, l, l^{\prime}\right) \leq O\left(n^{2} \log n\right)
$$

It is easy to see that this technique works for $\mathfrak{E}_{1}\left(k, k^{\prime}, l, l^{\prime}\right)$ in all other cases, and we get $\mathfrak{E}_{1} \leq O\left(n^{2} \log n\right)$ by (2.34).

$\mathfrak{E}_{2}$ is a quadruple sum over $(r, k),\left(r^{\prime}, k^{\prime}\right),(s, l),\left(s^{\prime}, l^{\prime}\right) \in B_{1}$ that is symmetric in $(r, k)$ and $(s, l)$ (as well as in $\left(r^{\prime}, k^{\prime}\right)$ and $\left.\left(s^{\prime}, l^{\prime}\right)\right)$. Recall that $V((r, k),(s, l))=0$ when $(r, k)=(s, l)$. If $(r, k) \notin\left\{\left(r^{\prime}, k^{\prime}\right),\left(s^{\prime}, l^{\prime}\right)\right\}$, then it is clear from independence that there is no contribution to $\mathfrak{E}_{2}$ on the event $\left\{X_{k} \neq(r, k)\right\}$. The contribution from the complementary event can be estimated using Lemma 2.7, just like in the case of $\mathfrak{E}_{1}$. 
Putting everything together and recalling (2.28), we see that

$$
\begin{aligned}
\mathfrak{E}_{2} \leq 2 E_{O}\left[\exp \{\cdots\} \sum_{\substack{(r, k) \in B_{1} \\
(s, l) \in B_{1}}}(V((r, k),(s, l))(a(\theta,(r, k))\right. \\
\left.-\mu \mathbb{1}_{\left\{X_{k}=(r, k)\right\}}\right)\left(a(\theta,(s, l))-\mu \mathbb{1}_{\left.\left.\left.\left\{X_{l}=(s, l)\right\}\right)\right)^{2}\right]+O\left(n^{2} \log n\right)}\right] \\
\leq 2(2|\theta|+\mu)^{4} E_{O}\left[\exp \left\{\left\langle\theta, X_{n}\right\rangle-n \log \phi(\theta)\right\} \sum_{\substack{(r, k) \in B_{1},(s, l) \in B_{1}}} V((r, k),(s, l))^{2}\right]+O\left(n^{2} \log n\right) \\
\leq 2(2|\theta|+\mu)^{4} 32 C_{1}^{2} C_{2}^{2} n^{2} \log n+O\left(n^{2} \log n\right) \\
\leq O\left(n^{2} \log n\right) .
\end{aligned}
$$

Finally,

$$
\begin{aligned}
& M_{n} E_{o}\left[\exp \left\{\left\langle\theta, X_{n}\right\rangle-n \log \phi(\theta)\right\}\left(D\left(B_{1}\right)-\mu^{2} v(n, X)\right)^{2}\right] \\
& \leq M_{n}\left(8 \mu^{2} \mathfrak{E}_{1}+2 \mathfrak{E}_{2}\right) \leq O\left((\log n)^{-1}\right)
\end{aligned}
$$

by (2.32) and (2.33). This concludes the proof of Theorem 1.6, apart from

Lemma 2.7. For any $z, z^{\prime} \in \mathcal{R}$,

$$
\sup _{x \in \mathbb{Z}^{3}}\left|\hat{P}_{z}^{\theta}\left(X_{m}=x\right)-\hat{P}_{z^{\prime}}^{\theta}\left(X_{m}=x\right)\right| \leq O\left(m^{-3 / 2}\right) \text { as } m \rightarrow \infty \text {. }
$$

Proof. Let $G^{\theta}$ be the centered Gaussian density on $\mathbb{R}^{2}$ that has the same covariance with $\left(\left\langle X_{1}, e_{1}\right\rangle,\left\langle X_{1}, e_{2}\right\rangle\right)$ under $\hat{P}_{o}^{\theta}$. For any $z \in \mathcal{R}$, it is shown in Theorem 22.1 of [2] that

$$
\begin{aligned}
& \sup _{x}\left|\hat{P}_{z}^{\theta}\left(X_{m}=x\right)-\frac{2}{m} G^{\theta}\left(\frac{\left\langle x-z-m \xi(\theta), e_{1}\right\rangle}{\sqrt{m}}, \frac{\left\langle x-z-m \xi(\theta), e_{2}\right\rangle}{\sqrt{m}}\right)\right| \\
& \leq O\left(m^{-3 / 2}\right) \text { as } m \rightarrow \infty .
\end{aligned}
$$

Here, the supremum is taken over all $x=\left(x_{1}, x_{2}, x_{3}\right) \in \mathbb{Z}^{3}$ such that $x_{1}+x_{2}+m+1$ is even and $x_{3}=m+1$. (Otherwise, $\hat{P}_{z}^{\theta}\left(X_{m}=x\right)$ is equal to zero.) Since $\sup _{y \in \mathbb{R}^{2}}\left|\nabla_{y} G^{\theta}(y)\right|<$ $\infty$, the desired result follows from the triangle inequality.

\section{Inequality of the Rate Functions for Space-Only RWRE}

3.1. Reducing to a fractional moment estimate. Consider space-only RWRE on $\mathbb{Z}^{d}$ with $d \geq 1$. Assume that the walk is non-nestling relative to the canonical basis vector $e_{d}$. By Jensen's inequality, the quenched and the averaged logarithmic moment generating functions 


$$
\begin{aligned}
& \Lambda_{q}(\theta):=\lim _{N \rightarrow \infty} \frac{1}{N} \log E_{o}^{\omega}\left[\exp \left\{\left\langle\theta, X_{N}\right\rangle\right\}\right] \text { and } \\
& \Lambda_{a}(\theta):=\lim _{N \rightarrow \infty} \frac{1}{N} \log E_{o}\left[\exp \left\{\left\langle\theta, X_{N}\right\rangle\right\}\right]
\end{aligned}
$$

satisfy $\Lambda_{q}(\theta) \leq \Lambda_{a}(\theta) \leq|\theta|$ for every $\theta \in \mathbb{R}^{d}$.

Recall the definition of regeneration times $\left(\tau_{n}\right)_{n \geq 0}$ (relative to $e_{d}$ ) given in Subsect. 1.2. Let

$$
\beta:=\inf \left\{i \geq 0:\left\langle X_{i}, e_{d}\right\rangle<\left\langle X_{o}, e_{d}\right\rangle\right\} \in[1, \infty]
$$

By the non-nestling assumption, there exist constants $c_{2}, c_{3}>0$ such that

$$
\operatorname{ess} \inf _{\mathbb{P}} P_{o}^{\omega}(\beta=\infty) \geq c_{2} \text { and } \operatorname{ess}_{\mathbb{P}} P_{o}^{\omega}\left(\tau_{1}>n\right) \leq \mathrm{e}^{-c_{3} n}
$$

for every $n \geq 1$, cf. [16]. These bounds clearly imply that

$$
\operatorname{ess} \sup _{\mathbb{P}} E_{o}^{\omega}\left[\exp \left\{c \tau_{1}\right\} \mid \beta=\infty\right] \leq c_{2}^{-1} \operatorname{ess} \sup _{\mathbb{P}} E_{o}^{\omega}\left[\exp \left\{c \tau_{1}\right\}\right]=: H(c)<\infty
$$

whenever $c<c_{3}$.

For every $c \in\left(0, c_{3}\right]$, introduce the set

$$
\mathcal{C}(c):=\left\{\theta \in \mathbb{R}^{d}: 2|\theta|<c\right\}
$$

Lemma 3.1. For every $\theta \in \mathcal{C}\left(c_{3}\right)$,

$$
E_{o}\left[\exp \left\{\left\langle\theta, X_{\tau_{1}}\right\rangle-\Lambda_{a}(\theta) \tau_{1}\right\} \mid \beta=\infty\right]=1 .
$$

$\Lambda_{a}$ is analytic on $\mathcal{C}\left(c_{3}\right) . \nabla \Lambda_{a}(0)=\xi_{o}$. The Hessian $\mathcal{H}_{a}$ of $\Lambda_{a}$ is positive definite on $\mathcal{C}\left(c_{3}\right)$. For every $c<c_{3}$ and $\theta \in \mathcal{C}(c)$, the smallest eigenvalue of $\mathcal{H}_{a}(\theta)$ is bounded from below by a positive constant that depends only on $c$ and the ellipticity constant $\kappa$ of the walk.

Proof. See the proofs of Lemmas 6 and 12 of [21]. In particular, the desired lower bound for the smallest eigenvalue of $\mathcal{H}_{a}$ is evident from Eq. (2.10) of that paper.

Given any $N \geq 1, \theta \in \mathcal{C}\left(c_{3}\right)$ and $\omega \in \Omega$, define

$$
\begin{aligned}
& \hat{W}_{N}(\theta, \omega):=E_{o}^{\omega}\left[\exp \left\{\left\langle\theta, X_{\tau_{N}}\right\rangle-\Lambda_{a}(\theta) \tau_{N}\right\}\right] \quad \text { and } \\
& W_{N}(\theta, \omega):=E_{o}^{\omega}\left[\exp \left\{\left\langle\theta, X_{\tau_{N}}\right\rangle-\Lambda_{a}(\theta) \tau_{N}\right\} \mid \beta=\infty\right]
\end{aligned}
$$

Lemma 3.2. For every $\theta \in \mathcal{C}\left(c_{3}\right)$, if

$$
\limsup _{N \rightarrow \infty} \frac{1}{N} \log \hat{W}_{N}(\theta, \cdot)<0
$$

holds $\mathbb{P}$-a.s., then $\Lambda_{q}(\theta)<\Lambda_{a}(\theta)$. 
Proof. Let $\theta \in \mathcal{C}\left(c_{3}\right)$. Then, $\theta \in \mathcal{C}(c)$ for some $c<c_{3}$. By hypothesis, for $\mathbb{P}$-a.e. $\omega$, there exist $C_{3} \geq 1$ and $c_{4}>0$ (both depending on $\omega$ ) such that $\hat{W}_{N}(\theta, \omega) \leq C_{3} \mathrm{e}^{-c_{4} N}$ for every $N \geq 1$.

Given any $n \geq 1$ and $K \geq 1$, it follows from Chebyshev's inequality and (3.2) that

$$
\begin{aligned}
& E_{o}^{\omega}\left[\exp \left\{\left\langle\theta, X_{n}\right\rangle-\Lambda_{a}(\theta) n\right\}\right] \\
& =E_{o}^{\omega}\left[\exp \left\{\left\langle\theta, X_{n}\right\rangle-\Lambda_{a}(\theta) n\right\}, n<\tau_{\left\lfloor\frac{n}{K}\right\rfloor}\right] \\
& +\sum_{j=\left\lfloor\frac{n}{K}\right\rfloor}^{n} E_{o}^{\omega}\left[\exp \left\{\left\langle\theta, X_{n}\right\rangle-\Lambda_{a}(\theta) n\right\}, \tau_{j} \leq n<\tau_{j+1}\right] \\
& \leq \mathrm{e}^{2|\theta| n} P_{o}^{\omega}\left(n<\tau_{\left\lfloor\frac{n}{K}\right\rfloor}\right)+\sum_{j=\left\lfloor\frac{n}{K}\right\rfloor}^{n} E_{o}^{\omega}\left[\exp \left\{\left\langle\theta, X_{\tau_{j}}\right\rangle-\Lambda_{a}(\theta) \tau_{j}\right\}\right] \\
& \times \operatorname{ess} \sup _{\mathbb{P}} E_{o}^{\omega^{\prime}}\left[\exp \left\{2|\theta| \tau_{1}\right\} \mid \beta=\infty\right] \\
& \leq \mathrm{e}^{(2|\theta|-c) n} E_{o}^{\omega}\left[\exp \left\{c \tau_{\left\lfloor\frac{n}{K}\right\rfloor}\right\}\right]+\sum_{j=\left\lfloor\frac{n}{K}\right\rfloor}^{n} \hat{W}_{j}(\theta, \omega) \operatorname{ess} \sup _{\mathbb{P}} E_{o}^{\omega^{\prime}}\left[\exp \left\{c \tau_{1}\right\} \mid \beta=\infty\right] \\
& \leq \mathrm{e}^{(2|\theta|-c) n} E_{o}^{\omega}\left[\exp \left\{c \tau_{1}\right\}\right]\left(\operatorname{ess} \sup _{\mathbb{P}} E_{o}^{\omega^{\prime}}\left[\exp \left\{c \tau_{1}\right\} \mid \beta=\infty\right]\right)^{\left\lfloor\frac{n}{K}\right\rfloor-1} \\
& +\sum_{j=\left\lfloor\frac{n}{K}\right\rfloor}^{n} \hat{W}_{j}(\theta, \omega) \operatorname{ess} \sup _{\mathbb{P}} E_{o}^{\omega^{\prime}}\left[\exp \left\{c \tau_{1}\right\} \mid \beta=\infty\right] \\
& \leq \mathrm{e}^{(2|\theta|-c) n} H(c)^{\left\lfloor\frac{n}{K}\right\rfloor}+H(c) \sum_{j=\left\lfloor\frac{n}{K}\right\rfloor}^{n} C_{3} \mathrm{e}^{-c_{4} j} .
\end{aligned}
$$

Take $K$ sufficiently large, and conclude that

$$
\Lambda_{q}(\theta)-\Lambda_{a}(\theta)=\lim _{n \rightarrow \infty} \frac{1}{n} \log E_{o}^{\omega}\left[\exp \left\{\left\langle\theta, X_{n}\right\rangle-\Lambda_{a}(\theta) n\right\}\right]<0 .
$$

Lemma 3.3. For every $\theta \in \mathcal{C}\left(c_{3}\right)$, if

$$
\limsup _{N \rightarrow \infty} \frac{1}{N} \log \mathbb{E}\left[W_{N}(\theta, \cdot)^{\alpha}\right]<0
$$

for some $\alpha \in(0,1)$, then $\Lambda_{q}(\theta)<\Lambda_{a}(\theta)$. Hence, by convex duality, $I_{a}<I_{q}$ at $\xi=\nabla \Lambda_{a}(\theta)$.

Proof. For any $N \geq 1$ and $\theta \in \mathcal{C}\left(c_{3}\right)$, it follows from the renewal structure and (3.4) that

$$
\begin{aligned}
\mathbb{E}\left[P_{o}^{\omega}(\beta=\infty) W_{N}(\theta, \cdot)\right] & =P_{o}(\beta=\infty) E_{o}\left[\exp \left\{\left\langle\theta, X_{\tau_{N}}\right\rangle-\Lambda_{a}(\theta) \tau_{N}\right\} \mid \beta=\infty\right] \\
& =P_{o}(\beta=\infty)\left(E_{o}\left[\exp \left\{\left\langle\theta, X_{\tau_{1}}\right\rangle-\Lambda_{a}(\theta) \tau_{1}\right\} \mid \beta=\infty\right]\right)^{N} \\
& =P_{o}(\beta=\infty) .
\end{aligned}
$$


Given any $\alpha \in(0,1)$, by the same reasoning as in (2.1),

$$
\limsup _{N \rightarrow \infty} \frac{1}{N} \log \hat{W}_{N}(\theta, \cdot) \leq \limsup _{N \rightarrow \infty} \frac{1}{N \alpha} \log \mathbb{E}\left[\hat{W}_{N}(\theta, \cdot)^{\alpha}\right], \quad \mathbb{P} \text {-a.s. }
$$

On the other hand, if $2|\theta|<c<c_{3}$, then we see by subadditivity, Chebyshev's inequality, and (3.2) that

$$
\begin{aligned}
\mathbb{E}\left[\hat{W}_{N+1}(\theta, \cdot)^{\alpha}\right] & =\mathbb{E}\left[\left(\sum_{x \in \mathbb{Z}^{d}} E_{o}^{\omega}\left[\exp \left\{\left\langle\theta, X_{\tau_{1}}\right\rangle-\Lambda_{a}(\theta) \tau_{1}\right\}, X_{\tau_{1}}=x\right] W_{N}\left(\theta, T_{x} \cdot\right)\right)^{\alpha}\right] \\
& \leq \mathbb{E}\left[\sum_{x \in \mathbb{Z}^{d}}\left(E_{o}^{\omega}\left[\exp \left\{\left\langle\theta, X_{\tau_{1}}\right\rangle-\Lambda_{a}(\theta) \tau_{1}\right\}, X_{\tau_{1}}=x\right]\right)^{\alpha} W_{N}\left(\theta, T_{x} \cdot\right)^{\alpha}\right] \\
& \leq \mathbb{E}\left[\sum_{x \in \mathbb{Z}^{d}}\left(E_{o}^{\omega}\left[\exp \left\{2|\theta| \tau_{1}\right\}, \tau_{1} \geq|x|_{1}\right]\right)^{\alpha} W_{N}\left(\theta, T_{x} \cdot\right)^{\alpha}\right] \\
& \leq \mathbb{E}\left[\sum_{x \in \mathbb{Z}^{d}}\left(\mathrm{e}^{(2|\theta|-c)|x|_{1}} E_{o}^{\omega}\left[\exp \left\{c \tau_{1}\right\}\right]\right)^{\alpha} W_{N}\left(\theta, T_{x} \cdot\right)^{\alpha}\right] \\
& \leq H(c)^{\alpha} \mathbb{E}\left[W_{N}(\theta, \cdot)^{\alpha}\right] \sum_{x \in \mathbb{Z}^{d}} \mathrm{e}^{(2|\theta|-c) \alpha|x|_{1}} .
\end{aligned}
$$

The desired result follows immediately from (3.6), (3.7) and Lemma 3.2.

3.2. The correlation condition. In this subsection, we will consider space-only RWRE on $\mathbb{Z}^{d}$ with $d=2,3$, assume that the walk is non-nestling relative to $e_{d}$, and outline how one can modify the arguments given in Sect. 2 in order to reduce (3.5) to a simpler inequality.

We start with $d=2$. For every $n \geq 1$ of the form $k^{2}$, and for every $y=\left(y^{\prime}, y^{\prime \prime}\right) \in \mathbb{Z}^{2}$, let

$$
J_{y}:=\left[\left(y^{\prime}-\frac{1}{2}\right) \sqrt{n},\left(y^{\prime}+\frac{1}{2}\right) \sqrt{n}\right) \times\left[\left(y^{\prime \prime}-\frac{1}{2}\right) \sqrt{n},\left(y^{\prime \prime}+\frac{1}{2}\right) \sqrt{n}\right) \subset \mathbb{R}^{2},
$$

cf. (2.3). Take $N=n m$ for some $m \geq 1$. For every $\theta \in \mathcal{C}\left(c_{3}\right), \omega \in \Omega$ and $Y=$ $\left(y_{1}, \ldots, y_{m}\right) \in\left(\mathbb{Z}^{2}\right)^{m}$, define

$$
\begin{gathered}
\bar{W}_{N}(\theta, \omega, Y):=E_{o}^{\omega}\left[\exp \left\{\left\langle\theta, X_{\tau_{N}}\right\rangle-\Lambda_{a}(\theta) \tau_{N}\right\}, X_{\tau_{j n}}-\lfloor j n \zeta(\theta)\rfloor \in J_{y_{j}}\right. \\
\quad \text { for every } j \leq m \mid \beta=\infty],
\end{gathered}
$$

cf. (2.4), where

$$
\zeta(\theta):=E_{o}\left[X_{\tau_{1}} \exp \left\{\left\langle\theta, X_{\tau_{1}}\right\rangle-\Lambda_{a}(\theta) \tau_{1}\right\} \mid \beta=\infty\right] .
$$

By subadditivity,

$$
\mathbb{E}\left[W_{N}(\theta, \cdot)^{\alpha}\right] \leq \sum_{Y} \mathbb{E}\left[\bar{W}_{N}(\theta, \cdot, Y)^{\alpha}\right]
$$


cf. (2.5). Given any $C_{1} \geq 1, Y=\left(y_{1}, \ldots, y_{m}\right) \in\left(\mathbb{Z}^{2}\right)^{m}$ and $j \in\{1, \ldots, m\}$, let

$$
\begin{aligned}
B_{j}=B_{j}\left(y_{j-1}, y_{j}\right):=\left\{(s, i) \in \mathbb{Z}^{2}:\right. & (j-1) n\left\langle\zeta(\theta), e_{2}\right\rangle+\sqrt{n}\left(y_{j-1}^{\prime \prime}+1 / 2\right) \\
& \leq i<j n\left\langle\zeta(\theta), e_{2}\right\rangle+\sqrt{n}\left(y_{j}^{\prime \prime}-1 / 2\right), \\
& \left.\left|\left(s-\sqrt{n} y_{j-1}^{\prime}\right)-\frac{\left\langle\zeta(\theta), e_{1}\right\rangle}{\left\langle\zeta(\theta), e_{2}\right\rangle}\left(i-\sqrt{n} y_{j-1}^{\prime \prime}\right)\right| \leq C_{1} \sqrt{n}\right\},
\end{aligned}
$$

cf. (2.7). Also, redefine $a(\theta, \cdot)$ by setting

$$
a(\theta, x):=\left\langle\theta, v\left(T_{x} \omega\right)\right\rangle-\mathbb{E}[\langle\theta, v(\cdot)\rangle]
$$

for every $x \in \mathbb{Z}^{2}$, where $v(\omega)=\sum_{z \in \mathcal{R}} \pi(0, z) z$ as before. Note that, under the assumptions stated in Definition 1.7, we have $\mathbb{E}[\langle\theta, v(\cdot)\rangle]=\left\langle\theta, \xi_{o}\right\rangle$. However, this equality does not necessarily hold in general.

With these modified definitions, the arguments in Subsects. 2.3 and 2.4 easily carry over, once one replaces the i.i.d. random variables

$$
E_{o}^{T_{X_{i}} \omega}\left[\exp \left\{\left\langle\theta, X_{1}\right\rangle-\log \phi(\theta)\right\}\right]
$$

by the variables

$$
E_{o}^{T_{X_{\tau_{i}}} \omega}\left[\exp \left\{\left\langle\theta, X_{\tau_{1}}\right\rangle-\Lambda_{a}(\theta) \tau_{1}\right\} \mid \beta=\infty\right]
$$

Therefore, in order to prove (3.5), it suffices to show that

$$
\begin{gathered}
\sum_{y \in \mathbb{Z}^{2}} \max _{x \in J_{o}} E_{x}\left[\exp \left\{\left\langle\theta, X_{\tau_{n}}-x\right\rangle-\Lambda_{a}(\theta) \tau_{n}+f_{K}\left(\delta_{n} D\left(B_{1}\right)\right)\right\},\right. \\
\left.X_{\tau_{n}}-\lfloor n \zeta(\theta)\rfloor \in J_{y} \mid \beta=\infty\right]^{\alpha}<1 / 2
\end{gathered}
$$

when $\zeta(\theta)$ is as in (3.8) and $n, K, C_{1}$ are sufficiently large, cf. Lemma 2.4. Here, $\alpha \in(0,1)$ is fixed, $f_{K}(u):=-K \mathbb{1}_{u \geq \mathrm{e}^{K^{2}}}$ and $\delta_{n}=C_{1}^{-1 / 2} n^{-3 / 4}$, as before.

We imitate (2.16), and write the sum in (3.9) as

$$
\left.\sum_{y \in \mathbb{Z}^{2}} \max _{x \in J_{o}} E_{x}[\cdots]^{\alpha}=\sum_{\substack{y \in \mathbb{Z}^{2}: \\|y|>R}} \max _{\substack{x \in J_{o} \\ E_{x}}} E_{x}[\cdots]^{\alpha}+\sum_{\substack{y \in \mathbb{Z}^{2}: \\|y| \leq R}} \max _{\substack{x \in J_{o} \\ E_{x}}} E_{x} \cdots\right]^{\alpha}
$$

with some large constant $R$, to be determined. Just like in the space-time case, the first sum on the RHS of (3.10) is bounded from above by

$$
\sum_{\substack{y \in \mathbb{Z}^{2}: \\|y|>R}} \hat{P}_{o}^{\theta}\left(\left|\frac{X_{n}-\lfloor n \zeta(\theta)\rfloor}{\sqrt{n}}\right| \geq|y|-1\right)^{\alpha} .
$$

Here, $\hat{P}_{o}^{\theta}$ is redefined to be the probability measure on paths induced by the random walk (in a deterministic environment) whose transition probabilities are given by

$$
q^{\theta}(x):=E_{o}\left[\exp \left\{\left\langle\theta, X_{\tau_{1}}\right\rangle-\Lambda_{a}(\theta) \tau_{1}\right\}, X_{\tau_{1}}=x \mid \beta=\infty\right], \quad x \in \mathbb{Z}^{2} .
$$


(Note that $\sum_{x \in \mathbb{Z}^{2}} q^{\theta}(x)=1$ by (3.4).) If $\hat{E}_{o}^{\theta}$ denotes the corresponding expectation, it is clear that

$$
\hat{E}_{o}^{\theta}\left[\exp \left\{c\left|X_{1}\right|\right\}\right]<\infty \quad \text { for every } c \in\left(0, c_{3}-2|\theta|\right) .
$$

Therefore, by Chebyshev's inequality, (3.11) can be made arbitrarily small (uniformly in large $n$ ) by choosing $R$ sufficiently large.

The second sum on the RHS of (3.10) can be controlled by showing that

$$
\begin{gathered}
\max _{\substack{y \in \mathbb{Z}^{2}: \\
|y| \leq R}} \max _{x \in J_{o}} E_{x}\left[\exp \left\{\left\langle\theta, X_{\tau_{n}}-x\right\rangle-\Lambda_{a}(\theta) \tau_{n}+f_{K}\left(\delta_{n} D\left(B_{1}\right)\right)\right\},\right. \\
\left.X_{\tau_{n}}-\lfloor n \zeta(\theta)\rfloor \in J_{y} \mid \beta=\infty\right]
\end{gathered}
$$

is small when $n, K$ and $C_{1}$ are sufficiently large. In the space-time case, the verification of the analogous statement, i.e., (2.18), relied on the fact that

$$
E_{o}\left[\exp \left\{\left\langle\theta, X_{n}\right\rangle-n \log \phi(\theta)\right\} \sum_{i=0}^{n-1} a\left(\theta, X_{i}\right)\right]
$$

grows linearly in $n$, cf. (2.21) and (2.22). In the space-only case, the drift vectors at the points off the path do not contribute to the mean of $D\left(B_{1}\right)$ under the tilted measure, and the drift vector at any point on the path contributes only once even if it is visited multiple times. Therefore, the statement concerning (3.14) needs to be replaced by the statement that

$$
E_{o}\left[\exp \left\{\left\langle\theta, X_{\tau_{n}}\right\rangle-\Lambda_{a}(\theta) \tau_{n}\right\} \sum_{x \in S\left(X, \tau_{n}\right)} a(\theta, x) \mid \beta=\infty\right]
$$

grows linearly in $n$. Here, for any $j \geq 1$,

$$
S(X, j):=\left\{X_{i}: 0 \leq i<j\right\} .
$$

In the space-time case, the variance of $D\left(B_{1}\right)$ under the tilted measure was shown to be $O\left(n^{3 / 2}\right)$ since the only non-vanishing terms were those corresponding to points $x, y \in \mathbb{Z}^{2}$ such that $x=y$. In the space-only case, steps of the walk between consecutive regeneration times are not independent, and we therefore need to also consider terms corresponding to $x$ and $y$ that are both on the path in the same regeneration block. However, since regeneration times have exponentially decaying tails, the total contribution of such terms is $O(n)$, and the variance of $D\left(B_{1}\right)$ under the tilted measure is still $O\left(n^{3 / 2}\right)$.

With these modifications, the argument in Subsect. 2.5 enables us to deduce (3.5) provided that (3.15) grows linearly in $n$. By the renewal structure, the latter is equivalent to the following correlation condition:

$$
\mu:=E_{o}\left[\exp \left\{\left\langle\theta, X_{\tau_{1}}\right\rangle-\Lambda_{a}(\theta) \tau_{1}\right\} \sum_{x \in S\left(X, \tau_{1}\right)} a(\theta, x) \mid \beta=\infty\right]>0 .
$$

(This replaces the choice of $\mu$ for the space-time case, see (2.24).)

For $d=3$, after modifying (3.13) by (i) taking the first maximum over $\left\{y \in \mathbb{Z}^{3}\right.$ : $|y| \leq R\}$, (ii) replacing the sets $J_{y}$ and $B_{1}$ by their three dimensional analogs, and (iii) redefining $D\left(B_{1}\right)$ as in (2.29), one can employ the reasoning above in order to reduce (3.5) to showing that (3.13) is small when $n, K$ and $C_{1}$ are sufficiently large. After that, 
one can set $\delta_{n}:=n^{-1}(\log n)^{-1 / 2}$, apply the same kind of modifications to the argument given in Subsect. 2.6, and further reduce (3.5) to (3.17). In particular, note that Lemma 2.7 continues to hold under the new definition of $\hat{P}_{o}^{\theta}$, thanks to (3.12). We omit the (routine) details.

We have arrived at the following theorem.

Theorem 3.4. Consider space-only $R W R E$ on $\mathbb{Z}^{d}$ with $d=2,3$. Assume (1.1), (1.3) and that the walk is non-nestling relative to $e_{d}$. Then, there exists an open set $\mathcal{A}_{\text {so }} \subset \mathbb{R}^{d}$ with the following properties:

(i) $I_{a}$ is strictly convex and analytic on $\mathcal{A}_{\text {so }}$,

(ii) $\xi_{o} \in \mathcal{A}_{\text {so }}$, and

(iii) for every $\xi \in \mathcal{A}_{\text {so }}$, the strict inequality $I_{a}(\xi)<I_{q}(\xi)$ holds if (3.17) is satisfied at $\theta:=\nabla I_{a}(\xi)$.

Proof. Recall (3.3), and define

$$
\mathcal{A}_{s o}:=\left\{\nabla \Lambda_{a}(\theta): \theta \in \mathcal{C}\left(c_{3}\right)\right\} .
$$

It follows from Lemma 3.1 and the inverse function theorem that $I_{a}$ is strictly convex and analytic on $\mathcal{A}_{s o}$ which is an open set containing $\xi_{o}$.

Take any $\xi \in \mathcal{A}_{s o}$. Note that $\theta:=\nabla I_{a}(\xi)$ satisfies $\xi=\nabla \Lambda_{a}(\theta)$ by convex duality. As outlined above, (3.17) implies (3.5). Hence, the desired result follows from Lemma 3.3.

3.3. Proof of Theorem 1.8. Consider space-only RWRE on $\mathbb{Z}^{d}$ with $d=2,3$. Fix a triple $p=\left(p^{+}, p^{o}, p^{-}\right)$of positive real numbers such that $p^{-}<p^{+}$and $p^{+}+p^{o}+p^{-}=1$. Assume that $\mathbb{P}$ is in class $\mathcal{M}_{\epsilon}(d, p)$ for some small $\epsilon>0$, cf. Definition 1.7. Assume that $\epsilon \leq \frac{p^{o}}{4(d-1)}$ so that the ellipticity constant $\kappa$ of the walk satisfies

$$
\kappa \geq \min \left(p^{+}, p^{-}, \frac{p^{o}}{4(d-1)}\right) .
$$

Lemma 3.5. There exist $C_{4} \geq 1$ and $c_{5}>0$ (depending only on $p$ ) such that $\mid \Lambda_{a}(\theta)-$ $\left.\left\langle\theta, \xi_{o}\right\rangle\left|\leq C_{4}\right| \theta\right|^{2}$ holds for every $\theta \in \mathcal{C}\left(c_{5}\right)$.

Proof. Recall (3.1). Note that $c_{3}$ depends only on the law of the regeneration times which, in turn, is determined by the fixed triple $p$. Moreover, the ellipticity constant $\kappa$ of the walk satisfies (3.18). Fix any $c_{5}<c_{3}$. The desired result follows immediately from Lemma 3.1.

Consider the set

$$
\mathcal{C}_{t}\left(c_{5}\right):=\left\{\theta \in \mathcal{C}\left(c_{5}\right):\left\langle\theta, e_{d}\right\rangle=0\right\}
$$

(Here, the subscript stands for transversal.) Take any $\theta \in \mathcal{C}_{t}\left(c_{5}\right)$. Recall the notation in (3.16). Since $\mathbb{P}$ is in class $\mathcal{M}_{\epsilon}(d, p)$, it is easy to see that

$$
\xi_{o}=\left(p^{+}-p^{-}\right) e_{d}, \quad\left\langle\theta, \xi_{o}\right\rangle=0, \quad \text { and } \quad|a(\theta, x)|=\left|\left\langle\theta, v\left(T_{x} \omega\right)\right\rangle\right| \leq 2 \epsilon(d-1)|\theta|
$$


for every $x \in \mathbb{Z}^{d}$. Similarly, the isotropy assumption ensures that

$$
Z(\theta)=Z\left(\theta, X, \tau_{1}, \omega\right):=\sum_{x \in S\left(X, \tau_{1}\right)} a(\theta, x)
$$

satisfies

$$
E_{o}[Z(\theta) \mid \beta=\infty]=E_{o}\left[\tau_{1} Z(\theta) \mid \beta=\infty\right]=0 .
$$

Our aim is to show that

$$
E_{o}\left[\exp \left\{\left\langle\theta, X_{\tau_{1}}\right\rangle-\Lambda_{a}(\theta) \tau_{1}\right\} Z(\theta) \mid \beta=\infty\right]>0
$$

for certain choices of $\theta$, to be determined later. Expanding the exponential on the LHS of (3.21), we see that

$$
\begin{aligned}
& E_{o}\left[\exp \left\{\left\langle\theta, X_{\tau_{1}}\right\rangle-\Lambda_{a}(\theta) \tau_{1}\right\} Z(\theta) \mid \beta=\infty\right] \\
& \quad \geq E_{o}\left[\left(1+\left\langle\theta, X_{\tau_{1}}\right\rangle-\Lambda_{a}(\theta) \tau_{1}\right) Z(\theta) \mid \beta=\infty\right]-C_{5}|\theta|^{3} \\
& \quad=E_{o}\left[\left\langle\theta, X_{\tau_{1}}\right\rangle Z(\theta) \mid \beta=\infty\right]-C_{5}|\theta|^{3} .
\end{aligned}
$$

Indeed, (3.22) follows from $|Z(\theta)| \leq 2 \epsilon(d-1)|\theta| \tau_{1}$ and

$$
\begin{aligned}
1+\left\langle\theta, X_{\tau_{1}}\right\rangle-\Lambda_{a}(\theta) \tau_{1} & \leq \exp \left\{\left\langle\theta, X_{\tau_{1}}\right\rangle-\Lambda_{a}(\theta) \tau_{1}\right\} \\
& \leq 1+\left\langle\theta, X_{\tau_{1}}\right\rangle-\Lambda_{a}(\theta) \tau_{1}+2|\theta|^{2} \tau_{1}^{2} \exp \left\{2|\theta| \tau_{1}\right\},
\end{aligned}
$$

$C_{5}$ is some constant that depends only on $p$ and $c_{5}$ and finally, (3.20) implies (3.23).

In order to estimate (3.23), we first provide a more convenient representation of the RWRE. Let $\left(b_{i}\right)_{i \geq 0}$ be an i.i.d. sequence of random variables taking values in $\left\{e_{d}, 0,-e_{d}\right\}$, with

$$
P\left(b_{1}=e_{d}\right)=p^{+}, \quad P\left(b_{1}=0\right)=p^{o}, \quad \text { and } \quad P\left(b_{1}=-e_{d}\right)=p^{-} .
$$

Let $\left(f_{i}\right)_{i \geq 0}$ be another i.i.d. sequence of random variables (independent of $\left.\left(b_{i}\right)_{i \geq 0}\right)$ taking values in the set $\left\{ \pm e_{j}: 1 \leq j<d\right\} \cup\{0\}$, with

$$
P\left(f_{1}=0\right)=\frac{2 \epsilon(d-1)}{p^{o}} \text { and } P\left(f_{1}= \pm e_{j}\right)=\frac{1}{2(d-1)}-\frac{\epsilon}{p^{o}} \quad \text { if } 1 \leq j<d .
$$

For any $\omega \in \Omega$, the walk $\left(X_{i}\right)_{i \geq 0}$ under $P_{o}^{\omega}$ can be constructed by setting

$$
X_{i+1}-X_{i}:=b_{i}+\left(1-\left|b_{i}\right|\right) f_{i}+\left(1-\left|b_{i}\right|\right)\left(1-\left|f_{i}\right|\right) U_{i},
$$

where $\left(U_{i}\right)_{i \geq 0}$ is a sequence of independent random variables taking values in $\left\{ \pm e_{j}\right.$ : $1 \leq j<d\}$, with

$$
P^{\omega}\left(U_{i}= \pm e_{j} \mid \mathcal{F}_{i}\right)=\frac{\pi\left(X_{i}, X_{i} \pm e_{j}\right)-\left(\frac{p^{o}}{2(d-1)}-\epsilon\right)}{2 \epsilon(d-1)} .
$$

Here, $\mathcal{F}_{i}=\sigma\left(X_{1}, \ldots, X_{i}\right)$. Note that the laws of the sequences $\left(b_{i}\right)_{i \geq 0}$ and $\left(f_{i}\right)_{i \geq 0}$ do not depend on the environment, and that $\tau_{1}$ is a function of $\left(b_{i}\right)_{i \geq 0}$ only. 
Let

$$
N_{i}:=\sum_{j=0}^{i-1} \mathbb{1}_{1=\left(1-\left|b_{i}\right|\right)\left(1-\left|f_{i}\right|\right)}
$$

Introduce the events $L_{0}:=\left\{N_{\tau_{1}}=0\right\}, L_{1}:=\left\{N_{\tau_{1}}=1\right\}$, and $L_{2}:=\left\{N_{\tau_{1}} \geq 2\right\}$. Let $\mathcal{G}:=\sigma\left(\left(b_{i}, f_{i}\right)_{i \geq 0}\right)$. Note that the events $L_{0}, L_{1}$ and $L_{2}$ are $\mathcal{G}$-measurable, and so is the event $\{\beta=\infty\}$. On the event $L_{0}$, the walker never sees the environment until $\tau_{1}$, and thus $X_{\tau_{1}}$ is $\mathcal{G}$-measurable. Also, for any $i \geq 0$, on the event $\left\{X_{i} \notin S(X, i)\right\}$ (i.e., when $X_{i}$ is a fresh point), $a\left(\theta, X_{i}\right)$ is independent of $\mathcal{F}_{i}$ and $\mathcal{G}$ under $P_{o}$. Therefore, by isotropy,

$$
E_{o}[Z(\theta) \mid \mathcal{G}]=E_{o}\left[\sum_{i=0}^{\tau_{1}-1} a\left(\theta, X_{i}\right) \mathbb{1}_{X_{i} \notin S(X, i)} \mid \mathcal{G}\right]=0 .
$$

Putting these observations together, we see that

$$
E_{o}\left[\left\langle\theta, X_{\tau_{1}}\right\rangle Z(\theta), L_{0}, \beta=\infty\right]=E_{o}\left[\left\langle\theta, X_{\tau_{1}}\right\rangle E_{o}[Z(\theta) \mid \mathcal{G}], L_{0}, \beta=\infty\right]=0 .
$$

On the other hand, it is easy to check that $P_{o}\left(L_{2}\right) \leq c_{6} \epsilon^{2}$ for some $c_{6}=c_{6}(p)$. By Hölder's inequality,

$$
\begin{aligned}
\left|E_{o}\left[\left\langle\theta, X_{\tau_{1}}\right\rangle Z(\theta), L_{2}, \beta=\infty\right]\right| & \leq P_{o}\left(L_{2}\right)^{2 / 3} E_{o}\left[\left|\left\langle\theta, X_{\tau_{1}}\right\rangle Z(\theta)\right|^{3}, \beta=\infty\right]^{1 / 3} \\
& \leq c_{7} \epsilon^{7 / 3}|\theta|^{2}
\end{aligned}
$$

for some $c_{7}=c_{7}(p)>0$. (Recall that $a(\theta, \cdot) \leq 2 \epsilon(d-1)|\theta|$, cf. (3.19).)

Finally, let $L_{1}^{\ell}=L_{1} \cap\left\{\left(1-\left|b_{\ell}\right|\right)\left(1-\left|f_{\ell}\right|\right)=1, \ell<\tau_{1}\right\}$. Then,

$$
E_{o}\left[\left\langle\theta, X_{\tau_{1}}\right\rangle Z(\theta), L_{1}, \beta=\infty\right]=\sum_{\ell=0}^{\infty} E_{o}\left[\left\langle\theta, X_{\tau_{1}}\right\rangle Z(\theta), L_{1}^{\ell}, \beta=\infty\right] .
$$

For every $\ell \geq 0$

$$
\begin{aligned}
E_{o}\left[\left\langle\theta, X_{\tau_{1}}\right\rangle Z(\theta), L_{1}^{\ell}, \beta=\infty\right]= & E_{o}\left[\left\langle\theta, X_{\ell}\right\rangle Z(\theta), L_{1}^{\ell}, \beta=\infty\right] \\
& +E_{o}\left[\left\langle\theta, X_{\ell+1}-X_{\ell}\right\rangle Z(\theta), L_{1}^{\ell}, \beta=\infty\right] \\
& +E_{o}\left[\left\langle\theta, X_{\tau_{1}}-X_{\ell+1}\right\rangle Z(\theta), L_{1}^{\ell}, \beta=\infty\right] .
\end{aligned}
$$

By computations similar to the one involving $L_{0}$, the first and the third terms on the RHS of (3.27) are zero. The second term is equal to

$$
\begin{aligned}
& E_{o}\left[\left\langle\theta, X_{\ell+1}-X_{\ell}\right\rangle\left\langle\theta, v\left(T_{X_{\ell}} \omega\right)\right\rangle, L_{1}^{\ell}, \beta=\infty\right] \\
& =P_{o}\left(L_{1}^{\ell}, \beta=\infty\right) \mathbb{E}\left[E^{\omega}\left[\left\langle\theta, U_{o}\right\rangle\right]\langle\theta, v(\omega)\rangle\right] \\
& =P_{o}\left(L_{1}^{\ell}, \beta=\infty\right) \mathbb{E}\left[\left(\sum_{z \neq \pm e_{d}} \frac{\pi(0, z)-\left(\frac{p^{o}}{2(d-1)}-\epsilon\right)}{2 \epsilon(d-1)}\langle\theta, z\rangle\right)\langle\theta, v(\omega)\rangle\right] \\
& =\frac{P_{o}\left(L_{1}^{\ell}, \beta=\infty\right)}{2 \epsilon(d-1)} \mathbb{E}\left[\langle\theta, v(\omega)\rangle^{2}\right] .
\end{aligned}
$$


Therefore, by (3.26),

$$
E_{o}\left[\left\langle\theta, X_{\tau_{1}}\right\rangle Z(\theta), L_{1}, \beta=\infty\right]=\frac{P_{o}\left(L_{1}, \beta=\infty\right)}{2 \epsilon(d-1)} \mathbb{E}\left[\langle\theta, v(\omega)\rangle^{2}\right] .
$$

It is easy to see that $P_{o}\left(L_{1}, \beta=\infty\right) \geq c_{8} \epsilon$ for some $c_{8}=c_{8}(p)>0$ if $\epsilon$ is small enough. Also, part (c) of Definition 1.7 ensures that $\mathbb{E}\left[\langle\theta, v(\omega)\rangle^{2}\right] \geq c_{9} \epsilon^{2}|\theta|^{2}$ for some $c_{9}=c_{9}(p)>0$. Hence,

$$
E_{o}\left[\left\langle\theta, X_{\tau_{1}}\right\rangle Z(\theta), L_{1}, \beta=\infty\right] \geq c_{10} \epsilon^{2}|\theta|^{2}
$$

for some $c_{10}=c_{10}(p)>0$. Combining (3.24), (3.25) and (3.28) gives

$$
\begin{aligned}
E_{o}\left[\left\langle\theta, X_{\tau_{1}}\right\rangle Z(\theta) \mid \beta=\infty\right]-C_{5}|\theta|^{3} & \geq c_{10} \epsilon^{2}|\theta|^{2}-c_{7} \epsilon^{7 / 3}|\theta|^{2}-C_{5}|\theta|^{3} \\
& =\left(\left(c_{10}-c_{7} \epsilon^{1 / 3}\right) \epsilon^{2}-C_{5}|\theta|\right)|\theta|^{2} .
\end{aligned}
$$

If $\epsilon<\left(c_{10} / c_{7}\right)^{3}$, then, for every $\theta \in \mathcal{C}_{t}\left(c_{5}\right)$ such that $0<|\theta|<\left(c_{10}-c_{7} \epsilon^{1 / 3}\right) \epsilon^{2} / C_{5}$,

$$
E_{o}\left[\exp \left\{\left\langle\theta, X_{\tau_{1}}\right\rangle-\Lambda_{a}(\theta) \tau_{1}\right\} Z(\theta) \mid \beta=\infty\right]>0
$$

by (3.23) and (3.29).

Finally, Theorem 3.4 implies that $I_{a}<I_{q}$ on the set

$$
\left\{\nabla \Lambda_{a}(\theta): \theta \in \mathcal{C}_{t}\left(c_{5}\right), 0<|\theta|<\left(c_{10}-c_{7} \epsilon^{1 / 3}\right) \epsilon^{2} / C_{5}\right\}
$$

whose closure contains the LLN velocity $\xi_{o}=\nabla \Lambda_{a}(0)$. We have proved Theorem 1.8 .

\section{Open Problems}

Our technique of proof puts several restrictions on the class of models treated. The following are natural questions we have not addressed.

(1) Does Theorem 1.8 extend to all space-only RWRE in dimension $d=2$, 3, or at least to those satisfying Sznitman's condition (T)? Note that, for non-nestling walks, it suffices to show that the correlation condition (3.17) is satisfied on a sequence $\left(\theta_{n}\right)_{n \geq 1}$ that converges to zero, cf. Theorem 3.4.

(2) In case $\sum \pi(0, z)\langle z, e\rangle$ is random for any $e \in \mathcal{R}_{\text {so }}$, is it true that $I_{q}(\xi)=I_{a}(\xi)$ only when $\xi=0$ or $I_{a}(\xi)=0$, as is the case in dimension $d=1$ ?

In our proof of Theorem 1.8 (specifically, in the proof of the correlation condition (3.17)), we used the isotropy assumption in order to get rid of a centering term under the (untilted) measure; this does not seem essential and probably, the lack of isotropy could be handled in the perturbative regime. However, getting rid of the perturbative restriction, or of the non-randomness in the $e_{d}$ direction, requires additional arguments.

Acknowledgements. This research was supported partially by a grant from the Israeli Science Foundation, and by the Alhadeff Fund at the Weizmann Institute. We thank Francis Comets for providing us with an update on polymer models and bringing the work of Lacoin [10] to our attention.

Open Access This article is distributed under the terms of the Creative Commons Attribution Noncommercial License which permits any noncommercial use, distribution, and reproduction in any medium, provided the original author(s) and source are credited. 


\section{References}

1. Berger, N.: Limiting velocity of high-dimensional random walk in random environment. Ann. Probab. 36(2), 728-738 (2008)

2. Bhattacharya, R.N., Ranga Rao, R.: Normal approximation and asymptotic expansions. Melbourne, FL: Robert E. Krieger Publishing Co. Inc., 1986, Reprint of the 1976 original

3. Comets, F., Gantert, N., Zeitouni, O.: Quenched, annealed and functional large deviations for one-dimensional random walk in random environment. Probab. Theory Related Fields 118(1), 65-114 (2000)

4. Dembo, A., Zeitouni, O.: Large deviations techniques and applications. Volume $\mathbf{3 8}$ of Applications of Mathematics (New York). New York: Springer-Verlag, Second edition, 1998

5. Derrida, B., Giacomin, G., Lacoin, H., Toninelli, F.L.: Fractional moment bounds and disorder relevance for pinning models. Commun. Math. Phys. 287(3), 867-887 (2009)

6. Giacomin, G., Lacoin, H., Toninelli, F.L.: Marginal relevance of disorder for pinning models. Commun. Pure Appl. Math. 63, 233-265 (2010)

7. Greven, A., den Hollander, F.: Large deviations for a random walk in random environment. Ann. Probab. 22(3), 1381-1428 (1994)

8. Grimmett, G.: Percolation, Volume 321 of Grundlehren der Mathematischen Wissenschaften [Fundamental Principles of Mathematical Sciences]. Berlin: Springer-Verlag, Second edition, 1999

9. Kesten, H.: A renewal theorem for random walk in a random environment. In: Probability (Proc. Sympos. Pure Math., Vol. XXXI, Univ. Illinois, Urbana, Ill., 1976), Providence, R.I.: Amer. Math. Soc., 1977, pp. 67-77

10. Lacoin, H.: New bounds for the free energy of directed polymers in dimension $1+1$ and $1+2$. Commun. Math. Phys. 294, 471-503 (2010)

11. Peterson, J., Zeitouni, O.: On the annealed large deviation rate function for a multi-dimensional random walk in random environment. ALEA 6, 349-368 (2009)

12. Rassoul-Agha, F.: Large deviations for random walks in a mixing random environment and other (nonMarkov) random walks. Comm. Pure Appl. Math. 57(9), 1178-1196 (2004)

13. Rassoul-Agha, F., Seppäläinen, T.: In preparation, 2009

14. Rassoul-Agha, F., Seppäläinen, T.: Process-level quenched large deviations for random walk in random environment. To appear in Ann. Inst. H. Poincaré Probab. Stat. 2009

15. Rosenbluth, J.: Quenched large deviations for multidimensional random walk in random environment: a variational formula. PhD thesis in Mathematics, New York University, 2006, http://arXiv.org/abs/0804. 1444vq[math.PR], 2008

16. Sznitman, A.-S.: Slowdown estimates and central limit theorem for random walks in random environment. J. Eur. Math. Soc. (JEMS) 2(2), 93-143 (2000)

17. Sznitman, A.-S.: On a class of transient random walks in random environment. Ann. Probab. 29(2), 724-765 (2001)

18. Sznitman, A.-S., Zerner, M.: A law of large numbers for random walks in random environment. Ann. Probab. 27(4), 1851-1869 (1999)

19. Toninelli, F.L.: Coarse graining, fractional moments and the critical slope of random copolymers. Electron. J. Probab. 14(20), 531-547 (2009)

20. Varadhan, S.R.S.: Large deviations for random walks in a random environment. Comm. Pure Appl. Math. 56(8), 1222-1245 (2003)

21. Yilmaz, A.: Averaged large deviations for random walk in a random environment. Ann. Inst. H. Poincaré Probab. Stat. 46(3), 853-868 (2010). doi:10.1214/09-AIHP332, 2009

22. Yilmaz, A.: Large deviations for random walk in a space-time product environment. Ann. Probab. 37(1), 189-205 (2009)

23. Yilmaz, A.: Quenched large deviations for random walk in a random environment. Comm. Pure Appl. Math. 62(8), 1033-1075 (2009)

24. Yilmaz, A.: Equality of averaged and quenched large deviations for random walks in random environments in dimensions four and higher. Probab. Theory Related Fields, 2010. doi:10.1007/s00440-010-0261-3, 2010

25. Zeitouni, O.: Random walks in random environments. J. Phys. A 39(40), R433-R464 (2006)

26. Zerner, M.P.W.: Lyapounov exponents and quenched large deviations for multidimensional random walk in random environment. Ann. Probab. 26(4), 1446-1476 (1998)

Communicated by F. Toninelli 